\title{
Solvencia exterior en los países de la Unión Europea
}

\author{
Vicente Donoso \\ Universidad Complutense e ICEI \\ Víctor Martín \\ Universidad Rey Juan Carlos e ICEI
}

\section{Resumen}

El presente trabajo analiza la solvencia exterior de los países de la Unión Europea-15 mediante el estudio de estacionariedad de las series de saldo por cuenta corriente. Para ello se aplican diversos tests de raices unitarias lineales, permitiendo la presencia de cambios estructurales en la tendencia estocástica de las series y cambios en el orden de integración. Asimismo, se contempla la posibilidad de que el saldo tenga un comportamiento no lineal. Sobre la base de esta metodología, se muestra que, a lo largo del periodo considerado (1960-2010), la situación de solvencia de los distintos países dista de ser uniforme y ha de ser cuidadosamente matizada.

Palabras clave: déficit exterior, solvencia exterior, tests de raices unitarias, cambio estructural, modelos no-lineales.

Clasificación JEL: C22, F32.

\section{Abstract}

In this paper we analyze the external solvency of the European Union-15 countries. For this purpose we test the current account stationarity by means of traditional unit root tests, taking into account the possibility of structural change and changes in the order of integration. Moreover we consider the possibility that the current account may follow a non-linear mean-reversion behaviour under the null of stationarity. Based on this methodology, we show that over the period 1960-2010, the external solvency of the various countries is far from uniform and has to be carefully clarified.

Keywords: external deficit, external solvency, unit root tests, structural change, non-linear models.

JEL classification: C22, F32.

\section{Introducción}

Hace todavía pocos años, con motivo de la euforia por la progresiva y exitosa implantación del euro y la constitución de la Unión Monetaria Europea, la preocupación por los problemas que podrían derivarse de la aparición de déficits sostenidos en la cuenta corriente de los países de la Unión, era prácticamente inexistente. Tanto que, entre los criterios fijados en Maastricht para ser admitido en la Eurozona, ninguno hacía referencia a las cuentas exteriores. La convicción vigente entre académicos y políticos era mayoritariamente que, en una zona de moneda única, tales déficits no tendrían más importancia que los que pudieran existir entre las provincias de un mismo país, por ejemplo, en Extremadura o Cataluña. 
La crisis, que se hace abierta e incuestionable hacia agosto de 2007, ha zarandeado tal visión optimista hasta el punto de que los déficits exteriores, sus efectos macroeconómicos y sobre las finanzas públicas, y su sostenibilidad, se han convertido en uno de los asuntos más recurrentes en la agenda de política económica y también, de forma progresiva, en la agenda de los académicos e investigadores. Explicar cómo se ha producido dicho cambio, cae fuera del ámbito de este trabajo. Damos por sentado que se ha producido y que la realidad ha desbaratado la visión optimista, derivada de dos premisas: las decisiones óptimas de los agentes privados y el funcionamiento perfecto de los mercados financieros en una economía global. Tales premisas parecieron correctas hasta el momento en que se descubrió el grave problema de los activos hipotecarios, quebró -como paradigma de una situación comprometida- el prestigioso banco Lehman-Brothers y se hizo efectiva la restricción crediticia. A partir de esos meses, la amplitud y persistencia de déficits asimétricos en la cuenta corriente de los países de la Unión-15 empujó a primer término el grave asunto de su sostenibilidad ${ }^{1}$.

El presente trabajo tiene por objeto analizar dicha cuestión, tanto desde un punto de vista más descriptivo como desde planteamientos econométricos. Para ello, el contenido se ha estructurado en los siguientes apartados: después de éste primero de introducción, en el segundo se describen los rasgos principales de la situación exterior de los 15 países más destacados de la Unión Europea; en el tercero se aborda el marco conceptual y analítico en el que se va a realizar el estudio; en el cuarto se resumen aquellos estudios empíricos que pueden considerarse antecedentes del que aquí se realiza; en el quinto se llevan a cabo los contrastes econométricos, desde dos enfoques distintos, el de las raíces unitarias lineales y el más reciente de raíces unitarias no lineales; finalmente, en el sexto se recogen las principales conclusiones y las posibles líneas de investigación futura.

\section{Situación presente y evolución}

Como se ha comentado en la introducción, el proceso de construcción de la Unión Económica y Monetaria se ha llevado a cabo bajo unas premisas que han resultado inciertas. Si no en la teoría, sí al menos en la política implementada, se daba por sentado que, con una única moneda que eliminaba el riesgo de tipo de cambio, y con perfecta movilidad de capitales, los desequilibrios exteriores no presentarían problema al ser fácilmente financiables. Por eso, entre las condiciones de Maastricht que han servido de filtro para el acceso a la Eurozona los criterios de convergencia nominal atendían a los aspectos referidos a la inflación, el tipo de interés, la estabilidad reciente de la moneda y, significativamente, al déficit y a la deuda públicas; pero, en cambio, no se pronunciaban sobre el comportamiento del sector

\footnotetext{
1 Véase un buen análisis descriptivo y una interesante interpretación en ARESTIS y PAÚL (2009).
} 
privado y no se prestaba atención a la posible divergencia -presente o futura-, en las cuentas exteriores. No puede negarse que, considerada en su conjunto, la situación exterior de la Unión Europea, medida por su cuenta corriente, daba pie para este optimismo, puesto que, el balance en cuenta corriente oscila claramente en torno al equilibrio, y el gradiente de variación se mueve entre $\pm 1,5$ por 100 del PIB de la UE15 (Gráfico 1).

\section{GRÁFICO 1}

SALDO DE LA CUENTA CORRIENTE: UNIÓN EUROPEA-15, 1960-2010. (PORCENTAJE DEL PIB)

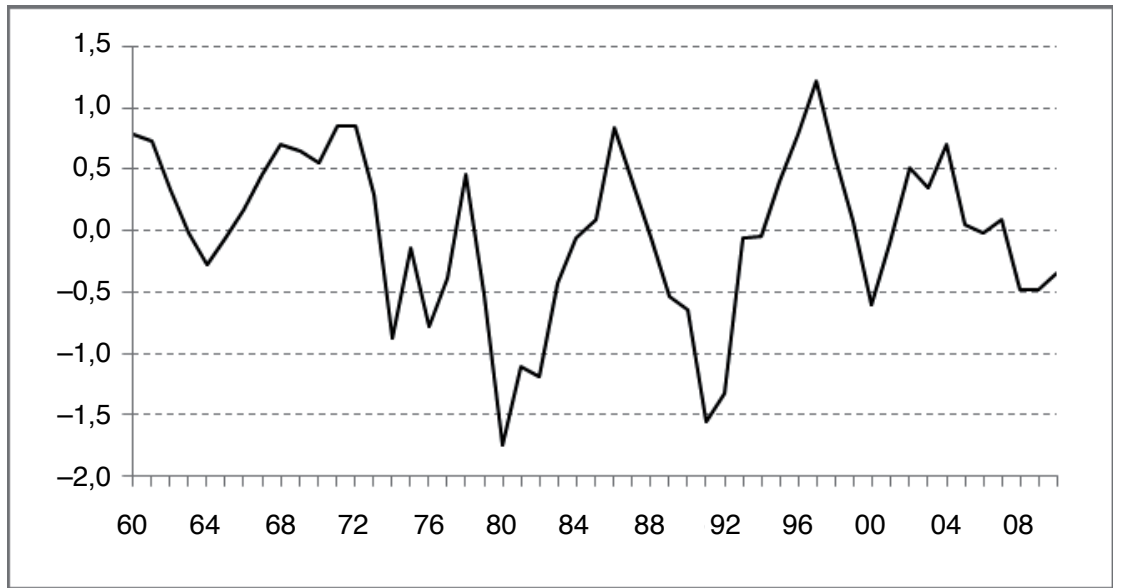

NOTA: A partir de 1991 incluye los datos de la Alemania Unificada. FUENTE: AMECO.

Sin embargo, frente a este optimismo de conjunto, la realidad de los diversos Estados miembros era bastante más matizada, si se atiende a la siguiente información empírica:

1. El perfil de los saldos en cuenta corriente (Gráfico 2) muestra en una amplía mayoría de Estados miembros una senda negativa, entre 1960 y 1991, antes de la cumbre de Maastricht, en la que se iban a fijar las condiciones. De hecho, como se recoge en el Cuadro 1, nueve de los quince miembros (Austria, Dinamarca, España, Finlandia, Francia, Grecia, Irlanda, Portugal y Reino Unido) tuvieron un promedio negativo a lo largo del citado período, con una horquilla que abarcó desde $-0,57$ del PIB del Reino Unido hasta el -5,50 del PIB de Portugal. Y entre los seis restantes, países como Bélgica, Italia y Suecia, arrojaron numerosos años de déficit, y un superávit promedio muy exiguo que apenas alcanzó el 0,5 del PIB. 
CUADRO 1

SALDO POR CUENTA CORRIENTE EN PORCENTAJE DEL PIB

\begin{tabular}{|c|c|c|c|c|c|c|}
\hline & \multicolumn{2}{|c|}{ 1960-2010 } & \multicolumn{2}{|c|}{ 1992-2010 } & \multicolumn{2}{|c|}{ 1960-1991 } \\
\hline & Media & d.t. & Media & d.t. & Media & d.t. \\
\hline Alemania & 1,51 & 2,40 & 1,89 & 3,25 & 1,28 & 1,66 \\
\hline Austria & $-0,61$ & 2,09 & 0,34 & 2,50 & $-1,17$ & 1,55 \\
\hline Bélgica & 1,81 & 2,38 & 4,03 & 1,22 & 0,50 & 1,88 \\
\hline Dinamarca & $-0,76$ & 2,80 & 2,35 & 1,40 & $-2,61$ & 1,49 \\
\hline España & $-2,20$ & 2,92 & $-4,15$ & 3,08 & $-1,04$ & 2,10 \\
\hline Finlandia & 0,12 & 3,76 & 3,80 & 3,25 & $-2,06$ & 1,86 \\
\hline Francia & $-0,62$ & 1,52 & $-0,12$ & 1,81 & $-0,91$ & 1,22 \\
\hline Grecia & $-3,56$ & 5,13 & $-8,04$ & 5,78 & $-0,91$ & 1,77 \\
\hline Holanda & 3,46 & 2,47 & 5,67 & 1,85 & 2,15 & 1,76 \\
\hline Irlanda & $-2,74$ & 4,03 & $-0,31$ & 2,74 & $-4,18$ & 3,99 \\
\hline Italia & $-0,03$ & 1,94 & $-0,33$ & 2,05 & 0,15 & 1,85 \\
\hline Luxemburgo & 11,66 & 5,03 & 10,32 & 2,34 & 12,45 & 5,95 \\
\hline Portugal & $-6,58$ & 3,68 & $-8,40$ & 2,39 & $-5,50$ & 3,89 \\
\hline Reino Unido & $-1,03$ & 1,53 & $-1,81$ & 0,81 & $-0,57$ & 1,67 \\
\hline Suecia & 1,84 & 3,11 & 4,91 & 2,88 & 0,02 & 1,23 \\
\hline
\end{tabular}

FUENTE: Elaboración propia a partir de AMECO, Comisión Europea.

2. Incluso antes del establecimiento de los criterios de Maastricht (1993) y de la puesta en funcionamiento de la Eurozona (1999), había claros indicios de desigualdad en los comportamientos exteriores. Una lectura atenta de estos indicios debería haber movido a considerar criterios de convergencia exterior antes de adentrarse en la uniformidad de la Unión Monetaria. Como se aprecia en el Cuadro 2, existía ya una clara heterogeneidad en el interior de la UE-15 en lo que respecta a los resultados de su cuenta corriente, es decir, en lo que respecta a la capacidad/necesidad de financiación relativa de los países. Un grupo de países (Alemania, Holanda y Suecia, a los que se pueden sumar Bélgica, Francia, Italia y Reino Unido) o no han tenido nunca, o sólo en muy contadas ocasiones, déficits que se cuenten entre el 25 por 100 de los más elevados. Por el contrario otro grupo de países (Dinamarca, Finlandia, Irlanda y Portugal, con el posible añadido de España y Grecia) han contado con numerosos años de déficit por encima del 75 por 100 de los países de la propia UE-15. Lo dicho basta para corroborar la afirmación de que hubiera sido una lectura prudente de la realidad, más allá de afirmaciones teóricas con fundamento lógico, pero con poca base empírica, el haber prestado atención a las disparidades de la cuenta corriente exterior en cuanto posible fuente de problemas futuros de la Unión Económica y Monetaria. Desde luego existe 
una razón pragmática o de política económica, para este comportamiento, y es que, aquellas magnitudes que de forma directa (déficits y deuda públicos) caen bajo el control del Gobierno son más fáciles de vigilar que aquellas otras donde existe una fuerte intervención del sector privado, como son las que atañen al comercio y a las inversiones internacionales.

\section{CUADRO 2}

\section{DÉFICITS ELEVADOS EN LA UNIÓN EUROPEA ANTES DE 1993} (TRATADO DE MAASTRICHT)

\begin{tabular}{|c|c|c|}
\hline Países & N. ${ }^{\mathbf{O}}$ & Años \\
\hline Alemania & 0 & \\
\hline Austria & 8 & 19601961197719781980198119841991 \\
\hline Bélgica & 3 & 1979-1981 \\
\hline Dinamarca & 23 & 1960-1962 1966-1973 1975-1979 1982-1988 \\
\hline España & 10 & 1963 1965-1967 19751976 1989-1992 \\
\hline Finlandia & 17 & 1960-1962196419651966 1970-19751983 1989-1992 \\
\hline Francia & 4 & 1969198219871988 \\
\hline Grecia & 6 & 196419651968196919851986 \\
\hline Holanda & 0 & \\
\hline Irlanda & 22 & 1962-1965 1968-1974 1976-1986 \\
\hline Italia & 3 & 196319731974 \\
\hline Portugal & 30 & 1960-1968 1970-1972 1974-1991 \\
\hline Reino Unido & 4 & 1987-1990 \\
\hline Suecia & 0 & \\
\hline
\end{tabular}

NOTAS: Déficit elevado = déficit igual o mayor al percentil 75 .

No se ha tenido en cuenta Luxemburgo.

FUENTE: Elaboración propia a partir de AMECO, Comisión Europea.

\section{GRÁFICO 2}

SALDO DE LA CUENTA CORRIENTE EN LOS PAÍSES DE LA UE-15, 1960-2010 (PORCENTAJE DEL PIB)

Alemania

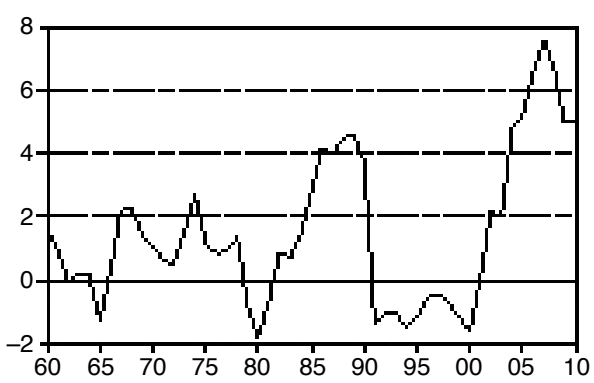

Austria

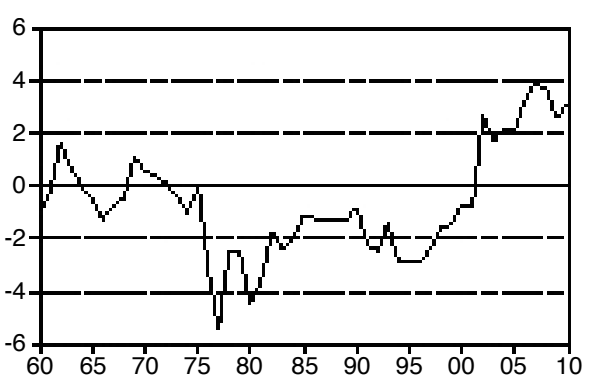




\section{GRÁFICO 2 (continuación)}

SALDO DE LA CUENTA CORRIENTE EN LOS PAÍSES DE LA UE-15, 1960-2010 (PORCENTAJE DEL PIB)
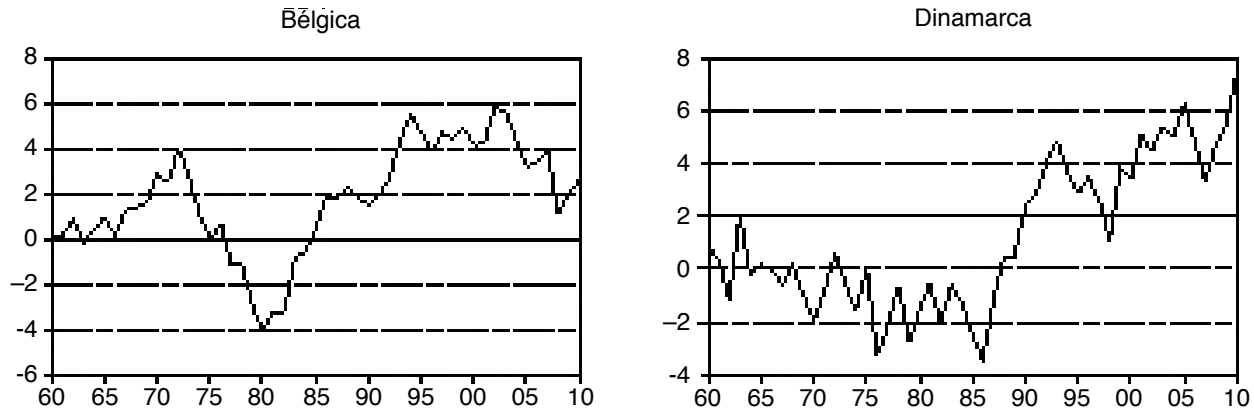

España

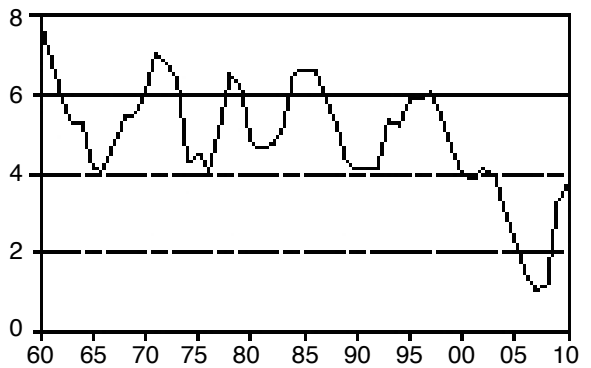

Finlandia

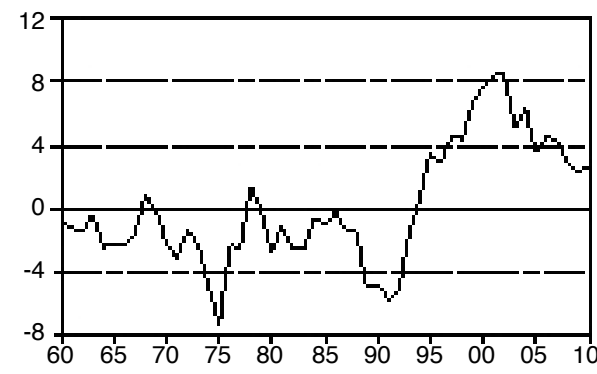

Francia

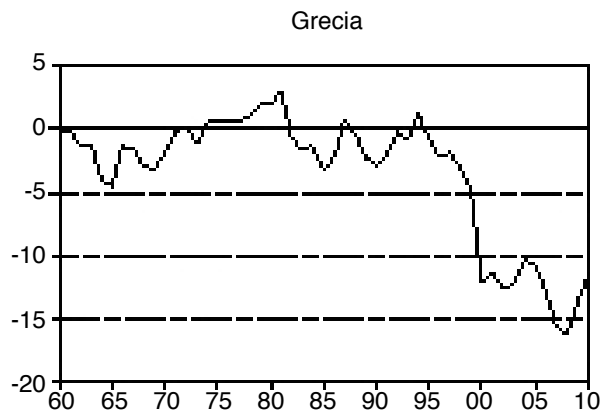

Holanda
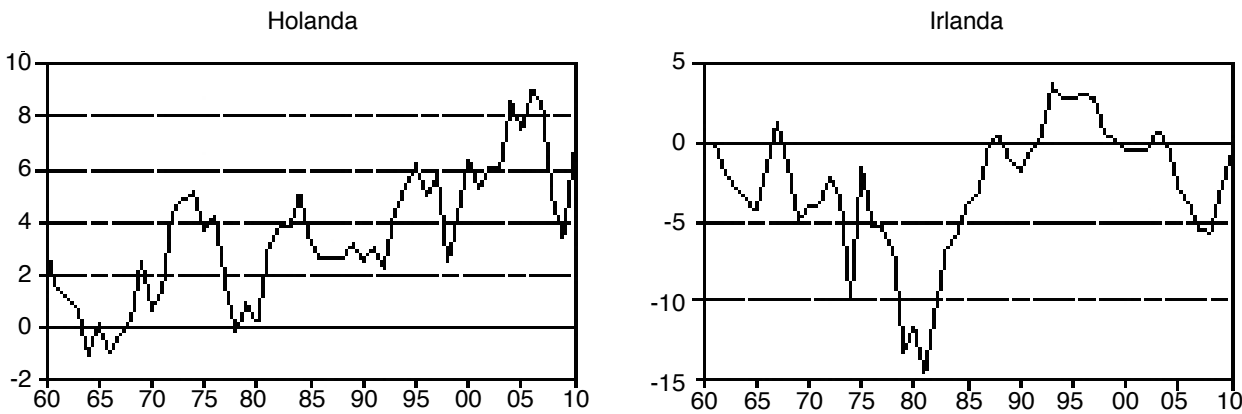
GRÁFICO 2 (continuación)

SALDO DE LA CUENTA CORRIENTE EN LOS PAÍSES DE LA UE-15, 1960-2010 (PORCENTAJE DEL PIB)

Italia

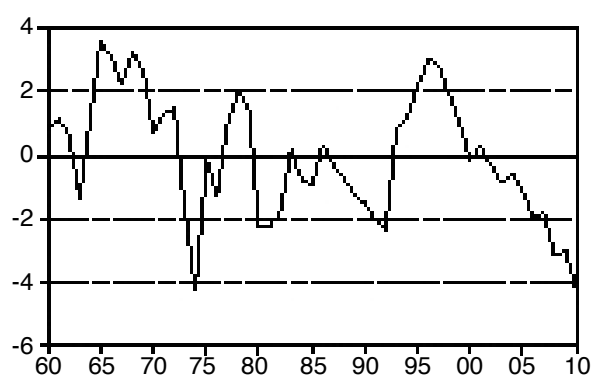

Portugal

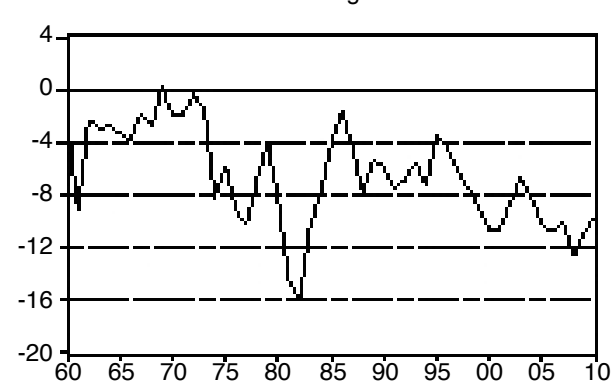

Luxemburgo

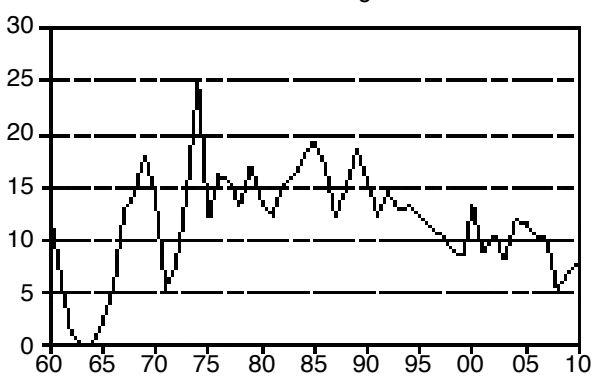

Suecia

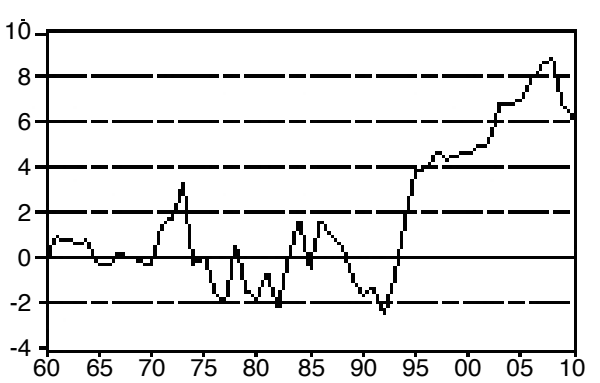

Reino Unido

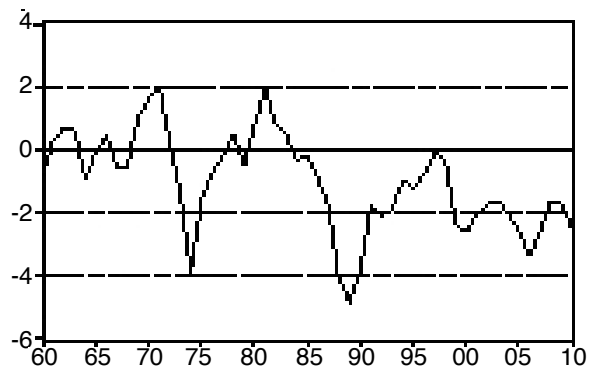


3. Por su relevancia y cercanía, la etapa a la que se debe prestar atención preferente es la que arranca en el entorno del Tratado de Maastricht, en 1993. Como es sabido, a partir de dicho año empieza el esfuerzo por aplicar los criterios de convergencia hasta culminar en 1999 con la implantación progresiva de la moneda única. Una nueva consulta a los gráficos representados en el Gráfico 2, muestra con suficiente claridad que, a comienzos de los años 90, se registran cambios importantes en las pautas de comportamiento de los distintos países. Uno primero concierne al aumento de la dispersión de los saldos intra-país cuando se compara el período 1960-1991 con el de 1992-2010: nueve países han visto incrementarse la desviación típica de sus saldos corrientes, lo que puede entenderse como un aumento de la sensibilidad de las cuentas exteriores frente a los cambios económicos (Cuadro 1). Otro dato apunta a que el promedio de los coeficientes de dispersión también aumenta entre ambos períodos, pasando del 2,26 al 2,49. Ahora bien, lo interesante para interpretar el momento presente es que esa mayor disparidad en los saldos exteriores puede atribuirse, de manera muy clara en la última década al comportamiento de España, Grecia y Portugal, según se comprueba en el Gráfico 3 .

\section{GRÁFICO 3}

\section{DISPERSIÓN EN EL SALDO POR CUENTA CORRIENTE EN LOS PAÍSES DE LA UE (DESVIACIÓN TÍPICA)}

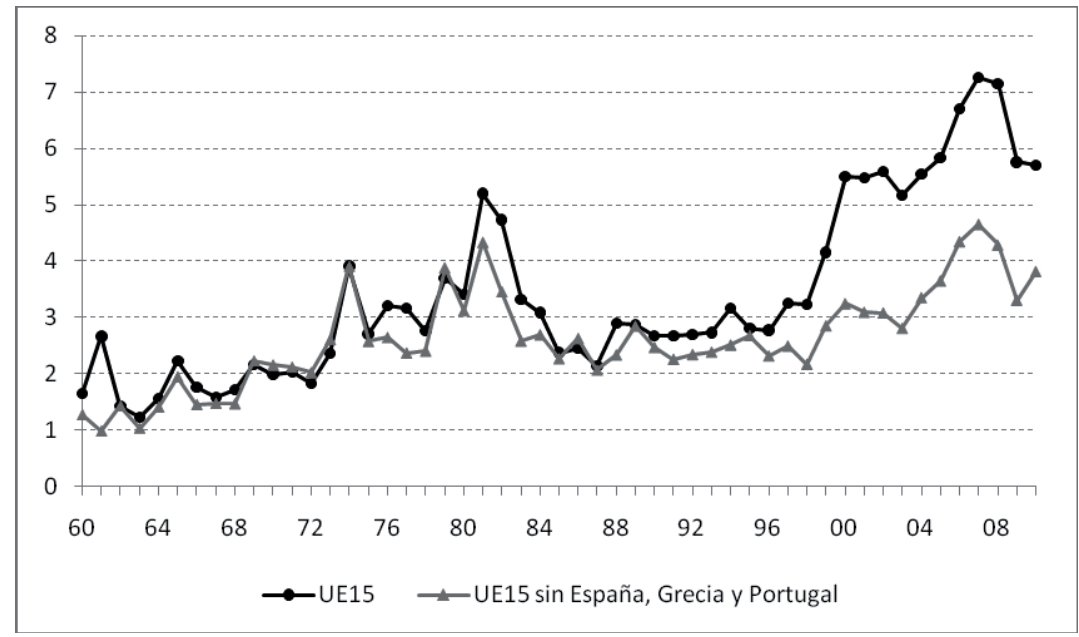

FUENTE: Elaboración propia a partir de AMECO, Comisión Europea. 
4. Los datos anteriores apuntan a un hecho importante que se ha ido acentuando desde el comienzo de la década de 1990, y es la progresiva polarización de los países de la UE-15 en dos bloques con resultados exteriores muy contrastados (Gráfico 2). Por una parte, están aquellos países que muestran un balance positivo en su cuenta corriente: Alemania, Austria, Bélgica, Dinamarca Finlandia, Holanda y Suecia. Pero, además, en estos países concurren otras circunstancias que otorgan a su economía una base firme en su evolución. La primera de ellas es el buen resultado en la balanza exterior de bienes y servicios en cuanto fundamento de una cuenta corriente positiva, y por tanto, de su capacidad de financiación al exterior; hay que destacar entre ellos, a Suecia, Holanda y Finlandia, con superávit por encima del 6 por 100 del PIB (Cuadro 3). La segunda circunstancia es su moderado nivel de deuda pública en relación al PIB, donde destaca el caso de Finlandia con un 46 por 100 , pero con porcentajes inferiores al 65 por 100 en el resto de los países mencionados; la excepción la constituye Bélgica con su abultadísimo 108 por 100, cifra que debe interpretarse a la luz de los otros datos económicos positivos (Cuadro 4). Por tanto, estos países concentran unas buenas perspectivas en su balance exterior, pero además, dada la competitividad que demuestran en su comercio, son compatibles con una política monetaria y de tipo de cambio del euro más bien estrictas; además, a juzgar por su relativamente bajo endeudamiento público promedio (con la excepción de Bélgica), no parece probable que experimenten tensiones en los mercados internacionales de deuda. En un contexto tan volátil y adverso, no puede garantizarse un futuro económico de estabilidad y crecimiento para ningún país; pero, por lo que se ha comentado, parece claro que los citados socios, miembros además de la Eurozona (a excepción de Dinamarca y Suecia) representan un espacio de mayor confianza y firmeza tanto para las finanzas de la Unión cuanto para la moneda única 
CUADRO 3

SALDO DE BIENES Y SERVICIOS EN PORCENTAJE DEL PIB

\begin{tabular}{|l|r|r|r|r|r|r|}
\hline \multirow{2}{*}{} & \multicolumn{2}{|c|}{$1960-2010$} & \multicolumn{2}{c|}{$\mathbf{1 9 9 2 - 2 0 1 0}$} & \multicolumn{2}{c|}{ 1960-1991 } \\
\cline { 2 - 7 } & Media & d.t. & Media & d.t. & Media & d.t. \\
\hline Alemania & 2,57 & 1,96 & 2,91 & 2,47 & 2,37 & 1,55 \\
Austria & 0,54 & 2,26 & 2,23 & 2,57 & $-0,46$ & 1,22 \\
Bélgica & 1,61 & 2,17 & 3,68 & 1,03 & 0,37 & 1,67 \\
Dinamarca & 1,08 & 3,54 & 4,77 & 1,38 & $-1,11$ & 2,44 \\
España & $-1,83$ & 2,17 & $-2,47$ & 2,24 & $-1,44$ & 2,02 \\
Finlandia & 1,88 & 3,79 & 6,02 & 2,46 & $-0,57$ & 1,76 \\
Francia & $-0,07$ & 1,38 & 0,34 & 1,60 & $-0,31$ & 1,17 \\
Grecia & $-7,19$ & 2,73 & $-9,55$ & 2,63 & $-5,78$ & 1,57 \\
Holanda & 3,44 & 2,86 & 6,30 & 1,38 & 1,75 & 2,03 \\
Irlanda & 1,90 & 9,63 & 12,66 & 3,01 & $-4,50$ & 5,72 \\
Italia & 0,52 & 1,70 & 1,27 & 1,83 & 0,08 & 1,45 \\
Luxemburgo & 10,41 & 10,61 & 21,81 & 8,20 & 3,64 & 4,07 \\
Portugal & $-9,41$ & 2,91 & $-8,82$ & 1,34 & $-9,76$ & 3,47 \\
Reino Unido & $-0,86$ & 1,69 & $-1,76$ & 1,20 & $-0,33$ & 1,71 \\
Suecia & 2,94 & 3,01 & 6,39 & 1,51 & 0,89 & 1,33 \\
\hline
\end{tabular}

FUENTE: Elaboración propia a partir de AMECO, Comisión Europea.

CUADRO 4

DEUDA PÚBLICA BRUTA EN PORCENTAJE DEL PIB

\begin{tabular}{|c|c|c|c|c|c|c|}
\hline & \multicolumn{2}{|c|}{ 1960-2010 } & \multicolumn{2}{|c|}{ 1992-2010 } & \multicolumn{2}{|c|}{ 1960-1991 } \\
\hline & Media & d.t. & Media & d.t. & Media & d.t. \\
\hline Alemania & 60,12 & 10,15 & 61,21 & 9,22 & - & - \\
\hline Austria & 64,26 & 4,23 & 65,10 & 3,50 & 56,24 & 0,09 \\
\hline Bélgica & 110,35 & 16,32 & 108,66 & 16,26 & 126,41 & 0,68 \\
\hline Dinamarca & 54,16 & 14,74 & 53,29 & 15,23 & 62,41 & 0,41 \\
\hline España & 52,69 & 9,53 & 53,71 & 9,46 & 43,02 & 0,39 \\
\hline Finlandia & 43,37 & 10,61 & 46,02 & 6,99 & 18,18 & 4,12 \\
\hline Francia & 46,91 & 17,72 & 60,46 & 9,51 & 28,52 & 5,44 \\
\hline Grecia & 99,83 & 15,28 & 102,74 & 13,00 & 72,19 & 1,16 \\
\hline Holanda & 62,66 & 11,23 & 61,18 & 10,79 & 76,71 & 0,14 \\
\hline Irlanda & 58,72 & 26,59 & 55,03 & 25,25 & 93,82 & 0,69 \\
\hline Italia & 104,66 & 12,70 & 111,65 & 6,44 & 88,04 & 7,33 \\
\hline Luxemburgo & 7,53 & 3,47 & 7,86 & 3,48 & 4,37 & 0,32 \\
\hline Portugal & 59,64 & 11,01 & 60,18 & 11,44 & 54,51 & 1,19 \\
\hline Reino Unido & 46,23 & 10,89 & 47,57 & 10,59 & 33,45 & 0,19 \\
\hline Suecia & 55,53 & 12,24 & 55,53 & 12,24 & - & - \\
\hline
\end{tabular}

FUENTE: Elaboración propia a partir de AMECO, Comisión Europea. 
5. En el otro extremo, otro grupo de países de la Unión (pertenecientes todos ellos también a la Eurozona) contrastan en su presente y en sus perspectivas. Hay que nombrar, particularmente, a España, Grecia y Portugal, economías en las que se da una evolución muy adversa de su cuenta corriente, de manera muy especial desde su incorporación a la Eurozona. Podría matizarse con razón que la situación de España es claramente más favorable que la de los otros dos socios. Para empezar, porque su déficit corriente, en el promedio de 1992-2010, es la mitad del correspondiente a Grecia y Portugal (4 por 100 frente al 8 por 100, aproximadamente); distancia que aún se hace más amplia si consideramos aquellas partidas que son las más importantes y la base de la solidez de unas cuentas exteriores saneadas, como son las del comercio de bienes y servicios, puesto que el saldo adverso de España, en torno al -2,5 por 100 , resalta favorablemente frente al 9 por 100 del descubierto de los dos socios mencionados. Pero además, la distancia favorable a nuestro país se incrementa si se tiene en cuenta el endeudamiento público, donde el 53 por 100 sobre el PIB del promedio español mejora al de Portugal (60 por 100) y sobre todo al de Grecia (102 por 100). Lo dicho, sin embargo, no debe hacer que se olviden dos circunstancias: el acelerado crecimiento de la ratio de deuda pública española sobre el PIB, (que según las previsiones se situará en el 74 por 100 en 2012) y la adversa percepción de los mercados que han elevado el diferencial del bono español a 10 años, en relación con el bono alemán, por encima de los 300 puntos básicos.

6. Haciendo puente entre ambos grupos, hay que mencionar a otros países europeos que presentan rasgos menos definidos: Francia, Italia, Irlanda y Reino Unido. Si se consideran los datos entre 1992-2010, se observa que arrojan unos déficits en cuenta corriente muy cercanos al equilibrio, con la excepción moderada del Reino Unido (déficit del 1, 80 por 100 del PIB). Pero además, Francia, Italia y sobre todo Irlanda (superávit promedio del 12 por 100 del PIB) presentan saldos positivos en su balance de bienes y servicios (la excepción, de nuevo, es el Reino Unido con un déficit promedio inferior al 2 por 100) lo que indica que tienen capacidad de competir y por tanto de aprovecharse del posible rebote de la economía mundial. Pero, además, su nivel de endeudamiento público es medio bajo, en relación con otros socios europeos, si se exceptúa en este aspecto a Italia, cuya ratio de deuda es preocupante (111 por 100 del PIB) y convierte a su economía en vulnerable. La evolución posterior ha demostrado que tanto este país como sobre todo Irlanda, han despertado la desconfianza de los mercados hacia su solvencia futura y su capacidad de competir y de crecer. Hacia donde se decanten los otros (notablemente Francia y el Reino Unido, economías de gran peso en Europa) aún no está claro y dependerá mucho de la esperada recuperación de la economía mundial. 
7. La situación que se ha descrito dibuja un horizonte de dificultades en el seno de la Unión Europea y más específicamente en el seno de la Eurozona. Existen varias razones de peso para ello:

a) La fecha de la recuperación mundial parece incierta, lo que perjudica a un espacio económico como la UE-15 que tiene en el comercio exterior uno de los pilares más fuertes de su crecimiento. Pero sin crecimiento, los problemas sociales (como el desempleo) seguirán pesando sobre el gasto público y, por el contrario, los ingresos disminuirán su participación en el producto. El resultado será más presión sobre los mercados de deuda pública y probablemente un aumento de las necesidades financieras de los agentes privados.

b) La polarización de situaciones, de la que se ha dado cuenta en los párrafos anteriores, dificulta una salida concertada de la crisis tanto en la Unión como en la Eurozona, puesto que las políticas necesarias no serán uniformes. Políticas de estímulo de la demanda, que probablemente favorezcan al primer grupo de países avanzados, por ejemplo, rebajando los tipos de interés al nivel de Japón y los Estados Unidos, y relajando la restricción monetaria, pueden desencadenar más inflación y deuda exterior en los países en dificultades, cuya cortedad competitiva puede impedirles aprovechar la coyuntura.

c) Esta heterogeneidad de situaciones, unida a la ausencia de una verdadera gobernanza, produce dos efectos perversos: retrasa la toma de decisiones (algunas urgentes para el futuro de la Eurozona), y merma la confianza, asunto particularmente importante cuando de lo que se trata es de defender la estabilidad del euro, uno de cuyos pilares, como en todo patrón fiduciario, es precisamente el mantenimiento de la confianza en la moneda. No necesita recordarse que la estabilidad de la Eurozona es fundamental para el conjunto de la Unión Europea.

\section{Marco conceptual}

El problema de la sostenibilidad del déficit exterior se ha convertido, de acuerdo con los datos empíricos que se han mostrado, en un tema de preocupación importante tanto para los responsables de la política económica cuanto para los académicos. Dicho problema presenta al menos dos características que le dotan de cierta dificultad $^{2}$. La primera es que se trata de un asunto complicado, es decir, de no fácil manejo, empezando por el ambiente de complacencia que ha habido hacia él en los ámbitos internacionales, concretamente de la Unión Europea y continuando por la

\footnotetext{
${ }^{2}$ Esto no significa que, en los tiempos recientes, no se hayan desarrollado procedimientos para analizar esta problemática desde sus diversos puntos de enfoque. Puede verse, por ejemplo, en relación al asunto de sostenibilidad exterior y el tipo de cambio: LEE et al. (2008).
} 
heterogeneidad de las situaciones en los diversos países. La segunda es que se trata de un asunto complejo, esto es, que presenta varias facetas diferentes aunque estén interrelacionadas. Para ganar claridad en los planteamientos analíticos y econométricos, conviene comentarlas.

En último término, la preocupación por el equilibrio externo se relaciona con el crecimiento. Quiere decirse que, desde un punto de vista económico, estamos interesados en analizar la situación exterior porque puede suponer una importante restricción al crecimiento. En los primeros planteamientos sobre este problema, el análisis se centró en los resultados de la cuenta comercial (exportación/importación de bienes y servicios), no considerando la existencia de otros saldos relacionados con las rentas internacionales o con las transferencias. Sin embargo, la falta de realismo de este supuesto ${ }^{3}$ (incluso antes de que la economía mundial se entrelazara y de que los movimientos de capital de todo tipo creciesen y con ellos los pagos por rentas), condujo a orientar el foco del análisis hacia el saldo de la cuenta corriente Un sencillo ejercicio de contabilidad nacional muestra que este saldo es igual a la diferencia entre el ahorro y la inversión, que a su vez se corresponde con la variación neta del stock de deuda internacional. Dicho saldo sirve para medir, en términos de contabilidad nacional, la capacidad o necesidad de financiación exterior.

Dentro de este marco de la balanza de pagos, donde queda identificado el equilibrio/desequilibrio exterior en relación al saldo de la cuenta corriente, la pregunta fundamental es: ¿cuando existe un problema de equilibrio externo que pueda afectar al crecimiento económico del país?

Lo primero que conviene subrayar es que los conceptos que emplean diversos autores están relacionados, pero no son coincidentes y presentan matices que son relevantes tanto en su utilidad de política como de análisis econométrico. Un buen punto de partida clarificador lo ofrece Milesi-Ferretti y Razin (1999). Dentro del marco planteado por estos autores, en el presente trabajo se distinguirá entre tres conceptos interrelacionados pero diferentes: la solvencia, la sostenibilidad y la cuantía excesiva del desequilibrio. Estas tres cuestiones responden al mismo problema, pero con tres matices distintos que son importantes a efectos sobre todo de la política económica. ¿Se podrá pagar el déficit?: pregunta por la solvencia; ¿se estará en condiciones de pagar el déficit?: pregunta por la sostenibilidad; ¿es excesivo el déficit?: pregunta por la cuantía concreta, en términos cardinales, o al menos en términos relativos al PIB ${ }^{4}$. En una economía real, las tres cuestiones están entrelazadas, pero a efectos de análisis conviene distinguirlas por sus diferentes implicaciones sobre la política económica.

En el presente trabajo se presta especial atención al problema de la solvencia. Se entiende que una economía es solvente si cumple la condición de que el valor pre-

\footnotetext{
3 En FERNÁNDEZ (2007), p. 8, puede encontrarse una clara demostración de que, con déficit comercial constante, el déficit por cuenta corriente, y con él la deuda externa, pueden crecer continuamente.

${ }^{4}$ Véanse, entre otros, MILESI-FERRETTI y RAZIN (1999) y FERNÁNDEZ CASTRO (2002).
} 
sente descontado de los superávit futuros de su cuenta corriente iguale el valor del sotck de su deuda externa neta; es decir, si cumple la restricción presupuestaria intertemporal (RPI). Se trata de una condición suficiente en el sentido de que el cumplimiento de la citada condición garantizaría que, bajo los supuestos y los datos empíricos aceptados, un país está en situación de hacer frente, con sus superávit futuros o esperados, a las obligaciones derivadas de su endeudamiento exterior. Ahora bien, en aquellos casos en que no se satisfaga la condición: existen dos posibles resultados de alcance muy distinto. Uno primero, que es sin duda el que en estos momentos más preocupa a Europa, es aquel en el que se demuestra que el país, bajo las circunstancias existentes, es insolvente porque los superávit esperados no serán capaces de absorber el montante de su deuda exterior neta. En tal situación pueden estar países como Grecia, Portugal, Italia y España, necesitados de políticas para recomponer sus cuentas exteriores. Otro segundo, que es menos preocupante, al menos desde el punto de vista del país en cuestión, es aquel en que resulta supersolvente, porque los ingresos netos futuros sobrepasan el valor de su stock de deuda externa neta. Este sería el caso, muy señalado, de economías como la japonesa o la alemana. Transformar la supersolvencia en un estado sostenible tiene la gran ventaja de su mayor facilidad y popularidad porque, en definitiva, se apoyaría en políticas de estímulo de la absorción interna, o dicho de otro modo, en la reducción del ahorro nacional, potenciando el consumo y la inversión.

Sin embargo, desde el punto de vista de la política económica, el cumplimiento de la citada condición de solvencia no garantiza que el país esté en condiciones reales de hacer frente, a largo plazo, a sus obligaciones financieras, es decir, que el endeudamiento sea sostenible. La citada condición sería una condición necesaria para la sostenibilidad, pero no una condición suficiente. Para que lo sea se requiere, además, o bien garantizar la continuidad de los supuestos actuales, frente a shocks aleatorios, caso de que la economía sea solvente; o bien introducir los cambios necesarios para que sea posible hacer efectiva la condición de solvencia. Estos cambios requieren circunstancias sociales y políticas que no siempre los gobiernos están en condiciones de garantizar, como se comprueba en la tenaz resistencia de las poblaciones de los estados en dificultades a los planes de austeridad. Si esto es así, los inversores, los mercados, descontarán este efecto e incrementarán las primas de riesgo empujando al país en cuestión hacia una situación insostenible. En este sentido, es de mucho interés el contrastar si se han dado, o se pueden dar, cambios estructurales que vayan a afectar de forma decisiva a las condiciones de solvencia, o lo que es lo mismo, a poner en cuestión la sostenibilidad del equilibrio exterior.

Por lo demás, instrumentar el análisis de la sostenibilidad resulta más complejo que el de la condición de solvencia, que tiene en la estacionariedad del saldo corriente una condición suficiente. Precisamente porque se requieren modelos estructurales, donde se incorporen variables que permitan captar la situación macro e incluso micro de ese país, y su efecto sobre la condición de solvencia. Estamos hablando de variables como ahorro e inversión, tasa de crecimiento, coeficiente de apertura externa, composición de los pasivos financieros...; y rasgos macroeconómicos como régimen 
de tipo de cambio, saldo fiscal, situación política... ${ }^{5}$. Reducir el análisis de sostenibilidad al cumplimiento de la condición de que la ratio del stock de deuda sobre el PIB sea constante, o lo que resulta equivalente, que la ratio del saldo de la cuenta corriente sobre el PIB sea constante, es sin duda una condición que, si se cumple, garantiza la solvencia, pero reduce notablemente el análisis de la sostenibilidad ${ }^{6}$.

Finalmente, la reflexión sobre el carácter excesivo o no del déficit tiene por objetivo establecer un límite cuantitativo al endeudamiento, en términos de sostenibilidad. Es decir, se trataría de cuantificar en términos absolutos o relativos al PIB un techo de déficit, más allá del cual, la reversión de los saldos resulta inevitable, o bien mediante un aterrizaje brusco provocado por la bancarrota, o bien mediante un aterrizaje suave, que provoque ajustes en el tipo de cambio, el consumo y la inversión ${ }^{7}$. Sobre esta cuestión cabe afirmar tres cosas. La primera es que, para poder establecerlo rigurosamente, hay que considerar que resulta intrínsecamente dependiente de las condiciones internas de cada país. La segunda es que las condiciones cuantitativas genéricas que han puesto en circulación Instituciones como el Banco Mundial, que hablan del 4 por 100 (otros estudiosos hablarían del 5 por 100) como límite prudente del déficit por cuenta corriente para prevenir un endeudamiento excesivo, no pasan de ser reglas empíricas que tan sólo pueden aspirar a orientar en sentido muy amplio la acción de la política económica, igual que en España la llamada regla del 4 por 100 pudo tener su utilidad a la hora de tomar decisiones que afectaban al cambio de la peseta ${ }^{8}$. La tercera es que el establecimiento riguroso de una condición cuantitativa de déficit y endeudamiento excesivo sólo puede llevarse a cabo mediante modelos de equilibrio general.

Sobre la base de los desarrollos anteriores, el presente trabajo se centrará en el análisis de la restricción presupuestaria intertemporal, teniendo en cuenta que su interpretación en términos de sostenibilidad o de magnitud excesiva hay que llevarla a cabo mediante la instrumentación de otras técnicas más complejas. Sin embargo, el conocimiento de las condiciones macro y microeconómicas de las economías analizadas permiten interesantes reflexiones sobre la materia.

\section{El criterio de estacionariedad de la cuenta corriente: estudios empíricos previos}

Desde mediados de la década de los ochenta y esencialmente a lo largo de la década de los noventa, los estudios empíricos que analizan la cuestión del déficit externo, se han basado en la aplicación de diversos métodos econométricos en el

\footnotetext{
5 Un amplio análisis en MILESI-FERRETTI y RAZIN (1999), pp. 21 y ss.

${ }^{6}$ Es lo que sucede en FERNÁNDEZ CASTRO (2002), pp. 56 y ss.

7 Véase, por ejemplo, CHRISTOPOULOS y LEÓN-LEDESEMA (2010).

8 Véanse CLARIDA et al. (2007), p.169; MILESI-FERRETTI y RAZIN (1999), p. 1; DONOSO y FARIÑAS (2011), p. 325.
} 
ámbito de las series temporales, con el fin de contrastar el cumplimiento de la restricción presupuestaria intertemporal (RPI) en diferentes economías. En términos de contabilidad nacional y según el método del gasto o de la demanda, la producción de bienes y servicios $\left(\mathrm{Y}_{\mathrm{t}}\right)$ de una economía abierta a lo largo de un periodo determinado «t» es la suma de cuatro componentes de manera que:

$$
Y_{t}=C_{t}+G_{t}+I_{t}+X_{t}-M_{t} \equiv C_{t}+G_{t}+I_{t}+X N_{t}
$$

en donde $C_{t}, G_{t}, I_{t}, X_{t}$ y $M_{t}$ son, respectivamente, consumo privado, gasto público, inversión, exportaciones de bienes y servicios e importaciones de bienes y servicios, $\mathrm{y}$ en donde $\mathrm{XN}_{\mathrm{t}}$ son exportaciones netas. Esta economía puede prestar y pedir prestados recursos al resto del mundo a un tipo de interés $r_{t}$, de forma que el balance por cuenta corriente a lo largo de «t» indica la variación en el stock de deuda externa de la economía entre dicho periodo $\left(\mathrm{B}_{\mathrm{t}}\right)$ y el periodo previo $\left(\mathrm{B}_{\mathrm{t}-1}\right)$,

$$
C A_{t}=B_{t}-B_{t-1}
$$

Asimismo la cuenta corriente puede expresarse como la suma de las exportaciones netas de bienes y servicios más el pago por intereses de la deuda externa tal que,

$$
C A_{t}=X N_{t}+r B_{t-1}
$$

Combinando [1.1], [1.2] y [1.3] tenemos que la restricción presupuestaria de la economía en cada periodo viene dada por,

$$
B_{t}-B_{t-1}=X N_{t}+r_{t} B_{t-1}=Y_{t}-C_{t}-G_{t}-I_{t}+r B_{t-1}
$$

Siguiendo el trabajo de Trehan y Wals (1991), es posible iterar la ecuación [1.4] hacia delante de manera que tomando valores esperados,

$$
B_{t-1}=-\sum_{j=0}^{\infty} R^{-(j+1)} E\left(X N_{t+j} / I_{t-1}\right)+\lim _{j \rightarrow \infty}\left[R ^ { - ( j + 1 ) } E \left(B_{t+j} / I_{t-1}\right.\right.
$$

en donde $I_{t}$ es el conjunto de información que poseen los agentes privados en el periodo $t$ y en donde $R_{t}=1+r_{t}$. Asimismo se supone que el tipo de interés $\left(r_{t}\right)$ es un proceso estocástico tal que $\mathrm{E}\left(\mathrm{r}_{\mathrm{t}+\mathrm{i}} / \mathrm{I}_{\mathrm{t}-1}\right)=\mathrm{r}$ para todo $\mathrm{i} \geq 0$. Cuando el último término en [1.5] se desvanece, el valor actual del stock de deuda externa coincide con la suma del valor descontado del saldo comercial futuro. De esta forma el cumplimiento de la RPI requiere que:

$$
\lim _{j \rightarrow \infty}\left[R^{-(j+1)} E\left(B_{t+j} / I_{t-1}\right)\right]=0
$$

condición que implica que el valor presente descontado del stock de deuda externa tiene que converger a cero a medida que «t» tiende a infinito. Trehan y Walsh (1991) 
demuestran que para el cumplimiento de la RPI de una economía con crecimiento nulo, es condición suficiente que el saldo por cuenta corriente sea un proceso estacionario. En el caso más realista en el que el producto o renta de la economía $\left(\mathrm{Y}_{\mathrm{t}}\right)$ crece a una tasa positiva no nula, la condición suficiente para que dicha condición se cumpla es que el ratio $\mathrm{CA}_{\mathrm{t}} / \mathrm{Y}_{\mathrm{t}}$ sea estacionario. De este modo, un déficit continuado es compatible con el cumplimiento de la RPI, siempre y cuando no crezca a una tasa mayor que la renta. En este caso la hipotésis de solvencia exterior implica que el saldo por cuenta corriente en porcentaje del PIB debe permanecer constante a largo plazo.

Desde el punto de vista empírico, se deduce de aquí una posible vía de contrastar la RPI del déficit exterior la cual consiste en analizar el orden de integración del saldo por cuenta corriente en porcentaje sobre el producto, mediante la aplicación de tests de raíces unitarias. En consecuencia, la estacionariedad del saldo por cuenta corriente en porcentaje del PIB, puede relacionarse con el concepto de solvencia exterior propuesto por Milesi-Ferreti y Razin (1999) tal que una economía es solvente si el valor presente descontado de los superávit futuros iguala el valor presente del stock de deuda externa neta. Los resultados que se obtienen de estos contrastes de estacionariedad ofrecen así una respuesta dicotómica al cumplimiento de la RPI, dándose la posibilidad de que se consideren como solventes (o no solventes) países cuya posición frente al exterior difiera notablemente entre sí, tanto en signo como en cuantía, al ser esas situaciones tan distintas compatibles con el criterio de estacionariedad de la cuenta corriente.

Los estudios empíricos que analizan el cumplimiento de la RPI son diversos. Gran parte de ellos se han centrado en el caso de los Estados Unidos, como por ejemplo Trehan y Walsh (1991), Husted (1992), Otto (1992), Wickens y Uctum (1993), Ahmed y Rogers (1995), Fisher (1995), Wu et al. (1996), Leachman y Francis (2000) y Christopoulos y León-Ledesma (2010). En los trabajos de Otto (1992) y Wu et al. (1996) se analiza además el caso de Canadá, mientras que Ahmed y Rogers (1995) analizan a su vez el caso del Reino Unido. Otros autores se centran en analizar la hipótesis de solvencia sobre un conjunto amplio de países desarrollados. Ejemplo de estos, es el trabajo de Liu y Tanner (1996), Dülger y Ozdemir (2005) y Chen (2011b) en donde se contrasta el cumplimiento de la RPI mediante la aplicación de diversos tests de raíces unitarias sobre el saldo por cuenta corriente de los países del G-7, o los trabajos de Gundlach y Sinn (1992) y Chen (2011ª) para el conjunto de países de la OCDE. Los resultados obtenidos en los distintos trabajos difieren aún cuando se analizan los mismos países, como consecuencia de la aplicación de diferentes metodologías y procedimientos, así como el uso de periodos maestrales distintos. En el Cuadro 5 se recogen las características de los principales estudios empíricos que analizan la solvencia exterior de alguno o varios países pertenecientes a la UE-15, así como los resultados sobre el cumplimiento o no de la RPI en los mismos. 
CUADRO 5

RESUMEN DE ESTUDIOS EMPÍRICOS PREVIOS

\begin{tabular}{|c|c|c|c|}
\hline Autores & Muestra & Metodología & RPI \\
\hline Anchuelo (1995) & España, 1967-1990 & Raíces unitarias & Se cumple \\
\hline $\begin{array}{l}\text { Apergis et al. } \\
(200)\end{array}$ & Grecia, 1969-1994 & $\begin{array}{l}\text { Cointegración } \\
\text { Gregory-Hansen }\end{array}$ & Se cumple \\
\hline Chen (2011a) & $\begin{array}{l}\text { Países de la OCDE: } \\
\text { Bélgica, Finlandia y } \\
\text { Portugal, 1975-2009 } \\
\text { España, 1983-2009 }\end{array}$ & $\begin{array}{l}\text { Raíces unitarias con } \\
\text { cambio de régimen }\end{array}$ & $\begin{array}{l}\text { Se cumple: Alemania } \\
\text { No se cumple: } \\
\text { Finlandia, Portugal } \\
\text { y España }\end{array}$ \\
\hline Chen (2011b) & $\begin{array}{l}\text { Países del G-7: } \\
\text { Alemania, 1991-2008 } \\
\text { Francia, 1975-2008 } \\
\text { Italia, 1971-2008 } \\
\text { Reino Unido, 1970-2008 }\end{array}$ & $\begin{array}{l}\text { Raíces unitarias con } \\
\text { cambio de régimen }\end{array}$ & $\begin{array}{l}\text { Se cumple: Alemania } \\
\text { No se cumple: } \\
\text { Francia, Italia } \\
\text { y Reino Unido }\end{array}$ \\
\hline $\begin{array}{l}\text { Dülger y Ozdemir } \\
(2005)\end{array}$ & Países del G-7, 1947-2001 & Integración fraccional & $\begin{array}{l}\text { Se cumple: Francia e Italia } \\
\text { No se cumple: Alemania } \\
\text { y Reino Unido }\end{array}$ \\
\hline $\begin{array}{l}\text { Gundlach y Sinn } \\
\text { (1992) }\end{array}$ & $\begin{array}{l}\text { Países de la OCDE, } \\
\text { 1950-1988 }\end{array}$ & Raíces unitarias & No se cumple: Alemania \\
\hline Holmes (2006) & $\begin{array}{l}\text { Países de la OCDE, } \\
\text { 1980-2002 }\end{array}$ & $\begin{array}{l}\text { Cointegración en datos } \\
\text { de panel }\end{array}$ & $\begin{array}{l}\text { Se cumple: } \\
\text { Bélgica y Reino Unido } \\
\text { No se cumple: Alemania, } \\
\text { Francia, Italia y España }\end{array}$ \\
\hline Kalyoncu (2006) & $\begin{array}{l}\text { Países de la OCDE, } \\
\text { 1960-2002 }\end{array}$ & $\begin{array}{l}\text { Raíces unitarias en datos } \\
\text { de panel }\end{array}$ & Se cumple \\
\hline $\begin{array}{l}\text { Liu y Tanner } \\
(1996)\end{array}$ & $\begin{array}{l}\text { Países del G-7, } \\
1970-1990\end{array}$ & $\begin{array}{l}\text { Raíces unitarias y } \\
\text { cambio estructural }\end{array}$ & $\begin{array}{l}\text { Se cumple: Alemania } \\
\text { No se cumple: Francia, } \\
\text { Italia y Reino Unido }\end{array}$ \\
\hline $\begin{array}{l}\text { Raibadui et al. } \\
\text { (2004) }\end{array}$ & $\begin{array}{l}\text { Argentina, Brasil, EE.UU., } \\
\text { Japón y Reino Unido } \\
(1970-2002)\end{array}$ & $\begin{array}{l}\text { Raíces unitarias con } \\
\text { cambio de régimen }\end{array}$ & $\begin{array}{l}\text { No se cumple: } \\
\text { Reino Unido } \\
:\end{array}$ \\
\hline Wu (2000) & Países de la OCDE & $\begin{array}{l}\text { Raíces unitarias en } \\
\text { datos de panel }\end{array}$ & Se cumple \\
\hline Wu et al. (2001) & $\begin{array}{l}\text { Países del G-7, } \\
\text { 1973-1998 }\end{array}$ & $\begin{array}{l}\text { Cointegración en datos } \\
\text { de panel }\end{array}$ & Se cumple \\
\hline
\end{tabular}

NOTAS: RPI: solo se muestran los resultados para los países de la UE-15.

FUENTE: Elaboración propia. 


\section{Contrastación empírica en la Unión Europea-15}

El análisis empírico que se ha realizado en el presente trabajo para comprobar el cumplimiento de la RPI en los países de la UE-15, tiene dos etapas: en primer lugar se estudia el orden de integración del saldo por cuenta corriente de cada una de las economías y se analiza la posible existencia tanto de cambios estructurales en la tendencia estocástica como en el orden de integración, todo ello en un contexto lineal; en segundo lugar se contrasta si las series presentan un comportamiento no lineal y se analiza el orden de integración del saldo por cuenta corriente en un contexto no lineal. Los datos empleados en el análisis son datos anuales del saldo por cuenta corriente en porcentaje del PIB para el periodo 1960-2010 procedentes de la base de datos AMECO elaborada por la Comisión Europea.

\subsection{Contrastes de raíz unitaria lineales y cambio estructural.}

Con el objetivo de analizar el orden de integración de las series de saldo por cuenta corriente $\left(\mathrm{CA}_{\mathrm{t}}\right)$ en porcentaje del PIB, se han aplicado dos contrastes de raíces unitarias lineales: el conocido test de raíces unitarias Dickey-Fuller Aumentado $^{9}$. (DFA), propuesto por Dickey y Fuller (1979) y Dickey y Fuller (1981) y el test Dickey-Fuller por Mínimos Cuadrados Generalizados (DF-MCG) desarrollado por Elliot et al. (1996) ${ }^{10}$ En ambos casos, los tests permiten contrastar la hipótesis nula de presencia de raíz unitaria. Si la hipótesis nula se rechaza se concluye que la serie en cuestión es estacionaria o integrada de orden cero $\mathrm{I}(0)$, lo que implicaría un saldo por cuenta corriente sostenible en el tiempo. Por el contrario, evidencia a favor de la hipótesis nula supone que la serie no es estacionaria y que al menos será integrada de orden uno, por lo que la evolución del saldo no es compatible con la hipótesis de solvencia. Los resultados se presentan en el Cuadro 6. En cada caso se muestran los resultados obtenidos para distintas especificaciones de los componentes deterministas del modelo. Sin embargo las conclusiones han de basarse principalmente en los resultados del modelo con constante, ya que en este caso, se estudia si el saldo por cuenta corriente presenta un comportamiento estacionario o no en torno a un valor medio distinto de cero.

\footnotetext{
9 Una breve descripción de este test, así como del resto de contrastes aplicados en el trabajo, puede encontrarse en el Anexo.

${ }^{10}$ Como demuestran los autores, el test DF-MCG presenta una mayor potencia que el test DFA en presencia de constante o tendencia lineal desconocida. Es decir, la probabilidad de rechazar la hipótesis nula siendo falsa es mayor.
} 
CUADRO 6

RESULTADOS DEL TEST DFA Y DF-MCG

\begin{tabular}{|l|c|c|c|c|c|}
\hline \multirow{2}{*}{} & \multicolumn{3}{|c|}{ DFA } & \multicolumn{2}{c|}{ DF-MCG } \\
\cline { 2 - 6 } & $\boldsymbol{\tau}$ & $\boldsymbol{\tau}_{\boldsymbol{\mu}}$ & $\boldsymbol{\tau}_{\boldsymbol{\tau}}$ & $\boldsymbol{\tau}_{\boldsymbol{\mu}}$ & $\boldsymbol{\tau}_{\boldsymbol{\tau}}$ \\
\hline Alemania & $-1,15$ & $-1,59$ & $-1,97$ & $-1,65$ & $-1,96$ \\
Austria & $-1,72$ & $-1,62$ & $-1,87$ & $-1,65$ & $-1,97$ \\
Bélgica & $-1,08$ & $-1,61$ & $-1,79$ & $-1,47$ & $-1,84$ \\
Dinamarca & $-0,76$ & $-0,48$ & $-2,91$ & $-0,61$ & $-2,58$ \\
España & $-2,01$ & $-2,94$ & $-3,64$ & $-1,85$ & $-3,48$ \\
Finlandia & $-1,69$ & $-1,68$ & $-2,36$ & $-1,68$ & $-2,25$ \\
Francia & $-1,36$ & $-1,81$ & $-1,82$ & $-1,17$ & $-1,77$ \\
Grecia & $-0,16$ & $-0,79$ & $-1,72$ & $-0,66$ & $-1,68$ \\
Irlanda & $-1,87$ & $-2,31$ & $-2,48$ & $-2,19$ & $-2,34$ \\
Holanda & $-0,93$ & $-2,11$ & $-4,01$ & $-2,13$ & $-3,57$ \\
Italia & $-2,48$ & $-2,45$ & $-2,89$ & $-2,46$ & $-2,99$ \\
Luxemburgo & $-1,25$ & $-2,96$ & $-2,92$ & $-2,99$ & $-2,99$ \\
Portugal & $-1,04$ & $-2,92$ & $-3,54$ & $-2,51$ & $-3,61$ \\
Suecia & $-0,70$ & $-1,20$ & $-2,17$ & $-1,07$ & $-2,11$ \\
Reino Unido & $-1,88$ & $-3,20$ & $-4,16$ & $-3,24$ & $-4,10$ \\
Valor crítico 5\% & $-1,95$ & $-2,92$ & $-3,50$ & $-1,95$ & $-3,19$ \\
\hline
\end{tabular}

NOTAS: $\tau=$ modelo sin componentes deterministas, $\tau_{\mu}=$ modelo con constante, $\tau_{\tau}=$ modelo con constante y tendencia. Valores críticos de MAcKINNON (1996). El número de retardos $(P)$ incluidos en cada estimación ha sido elegido mediante el criterio de información CS propuesto por SCHWARZ (1978).

FUENTE: Elaboración propia.

Como puede observarse, los únicos casos en los que se encuentra evidencia en contra de la hipótesis nula de presencia de raíz unitaria son España, Holanda, Luxemburgo, Portugal y Reino Unido. En el caso de Italia e Irlanda los resultados no son del todo concluyentes, pues tan sólo cuando se aplica el test DF-MCG permitiendo la presencia de un término constante, el estadístico toma un valor superior en términos absolutos al valor crítico correspondiente al 5 por 100 de significación. Ahora bien, estos resultados han de ser matizados si tenemos en cuenta que el periodo objeto de estudio está caracterizado por sucesivos cambios de política de tipo de cambio y otros eventos relevantes relacionados con el proceso de creación y la incorporación de los distintos países a la Unión Europea. Todo ello sugiere que el comportamiento del saldo por cuenta corriente de los países estudiados puede presentar cambios significativos a lo largo del periodo considerado, por lo que resulta interesante analizar la estacionariedad de las series permitiendo la presencia de cambios estructurales.

Con el fin de analizar la presencia de cambio estructural en la tendencia estocástica de la serie de saldo y variaciones en su orden de integración de forma endóge- 
na ${ }^{11}$, se ha seguido la metodología propuesta por Fernández (1999) y Fernández y Peruga (2005). Los resultados de los contrastes de cambio estructural en la tendencia estocástica se presentan en el Cuadro 7. Atendiendo a los valores del estadístico $\sup \left(\left|t_{\alpha}\right|\right)$ se observa que, en cuatro casos, la serie de saldo por cuenta corriente presenta un cambio estructural significativo: Dinamarca, Finlandia, Grecia y Suecia.

En el caso de Dinamarca el cambio aparece en el año 1987, coincidiendo con el inicio de una mejora significativa en el saldo corriente tras más de veinte años de déficit continuado. Dicha mejora, se produce principalmente como consecuencia de la consolidación fiscal iniciada en los años previos, así como de las diversas políticas aplicadas por el gobierno danés para estimular el ahorro privado y la demanda externa ${ }^{12}$. En Finlandia, el cambio estructural se produce en el año 1993, año en el que se empiezan a notar los primeros síntomas de mejora tras la depresión sufrida por la economía finlandesa a principios de la década de los años noventa, consecuencia de una fuerte crisis financiera y del colapso de la Unión Soviética, su principal mercado para las exportaciones ${ }^{13}$. A finales de 1991 el Banco de Finlandia devaluó la moneda del país en un 12 por 100 y en 1992 abandona el esquema cambiario de tipo de cambio fijo, lo que dio lugar a una severa depreciación del marco finlandés, cuyo valor frente al dólar se redujo en más de un 30 por 100. La consecuente ganancia de competitividad acompañada de un cambio en la estructura productiva del país, en donde los sectores de alta tecnología fueron ganando importancia relativa, fomentó el inicio de un proceso de crecimiento liderado por las exportaciones. Como reflejo, el déficit por cuenta corriente se reduce a partir de 1993 hasta alcanzar un saldo positivo superior al 8 por 100 del PIB en 2001.

En un contexto similar, puesto que la economía sueca también sufrió los efectos de una crisis financiera a principios de los años noventa, el cambio estructural del año 1994 en el caso de Suecia muestra una mejora notable del saldo corriente. Durante las décadas previas el saldo alterna periodos de superávit y déficits moderados, si bien a partir de 1994 la cuenta corriente mantiene siempre un resultado positivo.

11 Tal y como señalan CHRISTIANO (1992) y ZIVOT y ANDREWS (1992), la selección exógena de los puntos de cambio estructural reduce de forma considerable la potencia del contraste.

12 Ver FMI (1999).

13 Ver JONUNG y HAGBERG (2005). 
CUADRO 7

RESULTADOS DE LOS CONTRASTES DE CAMBIO ESTRUCTURAL

\begin{tabular}{|c|c|c|c|c|c|c|}
\hline & $\inf \left(t_{\rho}\right)$ & $\operatorname{media}\left(t_{\rho}\right)$ & $\sup \left(\left|t_{\alpha^{\prime}}\right|\right)$ & $\operatorname{media}\left(\left|t_{\alpha^{\prime}}\right|\right)$ & $\sup \left(\left|\mathbf{t}_{\left(\alpha^{\prime}\right)}\right|\right)$ & $\operatorname{media}\left(\left|t_{\left(\alpha^{\prime}\right)}\right|\right)$ \\
\hline \multirow[t]{2}{*}{ Alemania } & $-3,22$ & $-1,85$ & 3,17 & 1,07 & 1,69 & 0,67 \\
\hline & 2002 & - & 2001 & - & 2001 & - \\
\hline \multirow[t]{2}{*}{ Austria } & $-3,66$ & $-1,97$ & 3,38 & 1,20 & 1,61 & 0,68 \\
\hline & 2002 & - & 2002 & - & 1963 & - \\
\hline \multirow[t]{2}{*}{ Bélgica } & $-2,75$ & $-1,79$ & 2,52 & 0,82 & 1,43 & 0,54 \\
\hline & 1983 & - & 1983 & - & 1981 & - \\
\hline \multirow[t]{2}{*}{ Dinamarca } & $-3,47$ & $-1,40$ & 3,93 & 1,63 & 2,00 & 0,89 \\
\hline & 1988 & - & 1987 & - & 1987 & - \\
\hline \multirow[t]{2}{*}{ España } & $-4,86$ & $-3,39$ & 3,58 & 1,44 & 1,35 & 0,35 \\
\hline & 2003 & - & 2003 & - & 1966 & - \\
\hline \multirow[t]{2}{*}{ Finlandia } & $-4,31$ & $-2,18$ & 4,04 & 1,34 & 1,32 & 0,51 \\
\hline & 1993 & - & 1993 & - & 2003 & - \\
\hline \multirow{2}{*}{ Francia } & $-2,36$ & $-1,77$ & 2,21 & 0,59 & 2,04 & 0,68 \\
\hline & 2003 & - & 2003 & - & 2000 & - \\
\hline \multirow[t]{2}{*}{ Grecia } & $-4,87$ & $-1,78$ & 4,77 & 1,47 & 1,45 & 0,66 \\
\hline & 2000 & - & 2000 & - & 1995 & - \\
\hline \multirow[t]{2}{*}{ Holanda } & $-3,69$ & $-2,91$ & 2,98 & 2,01 & 1,44 & 0,44 \\
\hline & 1993 & - & 1993 & - & 1965 & - \\
\hline \multirow[t]{2}{*}{ Irlanda } & $-3,14$ & $-2,46$ & 2,52 & 0,82 & 1,61 & 0,46 \\
\hline & 1983 & - & 1982 & - & 1982 & - \\
\hline \multirow{2}{*}{ Italia } & $-3,11$ & $-2,67$ & 2,03 & 1,10 & 1,25 & 0,50 \\
\hline & 2003 & - & 2002 & - & 1998 & - \\
\hline \multirow[t]{2}{*}{ Luxemburgo } & $-4,67$ & $-3,21$ & 3,55 & 1,11 & 2,00 & 0,45 \\
\hline & 1967 & - & 1966 & - & 1963 & - \\
\hline \multirow[t]{2}{*}{ Portugal } & $-4,24$ & $-3,27$ & 2,88 & 1,34 & 1,03 & 0,25 \\
\hline & 1974 & - & 1974 & - & 1983 & - \\
\hline \multirow[t]{2}{*}{ Suecia } & $-4,38$ & $-1,95$ & 4,36 & 1,49 & 1,47 & 0,48 \\
\hline & 1994 & - & 1994 & - & 1993 & - \\
\hline \multirow[t]{2}{*}{ Reino Unido } & $-3,69$ & $-2,84$ & 2,67 & 1,41 & 1,04 & 0,40 \\
\hline & 1986 & - & 1986 & - & 1963 & - \\
\hline V. crítico $5 \%$ & $-4,38$ & $-2,92$ & 4,22 & 1,95 & 3,37 & 1,70 \\
\hline
\end{tabular}

NOTAS: Valores críticos de FERNÁNDEZ (1999, Tabla II.1, pp. 54-55, T = 50).

FUENTE: Elaboración propia.

La ruptura estructural en la evolución del saldo por cuenta corriente en Grecia se produce en el año 2000, un año antes de su incorporación a la UEM. Al contrario que en los tres casos previos, el cambio estructural refleja aquí un proceso de deterioro continuado del saldo corriente que se inicia a finales de la década de los años noventa, a partir del cual, y hasta la actualidad, el déficit por cuenta corriente se ha mantenido de forma sistemática por encima del 10 por 100 del PIB. Algunos autores coinciden en señalar los siguientes factores como causa principal del incremento del déficit ${ }^{14}$ : la pérdida gradual de la competitividad vía precios reflejo del dife-

\footnotetext{
14 Ver BITZIS et al.
} 
rencial de inflación y el incremento de los costes laborales unitarios respecto del resto de países de la zona euro; y el incremento de la demanda interna fruto de la liberalización financiera a mediados de los años noventa y la caída en el tipo de interés desde su incorporación a la UEM.

Cuando se tienen en cuenta estos cambios estructurales en la tendencia estocástica, los resultados del contraste de la hipótesis nula de presencia de raíz unitaria en Finlandia, Grecia y Suecia cambian. En estos tres casos el estadístico inf $\left(\mathrm{t}_{\rho 1}\right)$ toma, en términos absolutos, un valor superior al valor crítico correspondiente por lo que la evidencia es favorable a la hipótesis de estacionariedad del saldo por cuenta corriente.

Por su parte, los resultados de analizar la presencia de cambios en el orden de integración de las series se presentan en el Cuadro 8. Al 5 por 100 de significación, tan solo España y Portugal muestran cambios significativos. En estos dos casos, cuando se impone la presencia de raíz unitaria en la segunda parte de la muestra, los estadísticos $\inf \left(\mathrm{t}_{\rho 1}\right)$ y $\inf \left(\mathrm{t}_{\gamma 1}\right)$ coinciden en señalar el año 1999 y el año 1974 como las fechas en la que se produce un cambio en el orden de integración de la serie de saldo por cuenta corriente de España y Portugal respectivamente. En España los resultados sugieren que en el periodo previo a la introducción del euro en el sistema monetario español, el saldo por cuenta corriente presenta un comportamiento estacionario. A partir de 1999, sin embargo, dicho comportamiento se modifica. Atendiendo a la evolución del saldo (Gráfico 2) se observa que, hasta aproximadamente el año 1999 la serie deambula en torno a un valor medio del $-1,1$ por 100, reduciéndose a continuación de forma notable y continuada hasta el año 2008, año este en el que se produce una cierta mejora en el saldo corriente, consecuencia de la crisis financiera internacional.

Una posible causa de este comportamiento diferenciado del saldo en los periodos 1960-1998 y 1999-2010, es la pérdida del tipo de cambio como instrumento de ajuste de la competitividad exterior de la economía. Pues en efecto, en el primer periodo mencionado se suceden diversas depreciaciones y devaluaciones de la peseta, coincidiendo generalmente con periodos en los que el déficit por cuenta corriente se sitúa entre los tres y cuatro puntos porcentuales del PIB ${ }^{15}$ : entre 1975 y 1989 la peseta fue sometida al menos a tres depreciaciones frente al dólar, 1976:02 (12,8 por 100), 1977:07 (23,8 por 100) y 1982:12 (8 por 100); entre 1989 y 1995 sufrió cuatro devaluaciones frente al ECU, 1992:09 (5 por 100), 1992:11 (6 por 100), 1993:05 (8 por 100) y 1995:03 (5 por 100). Por su parte en Portugal, tan solo en el periodo comprendido entre 1960 y 1974, la evolución del saldo por cuenta corriente es compatible con el cumplimiento de la RPI.

\footnotetext{
15 Véase DONOSO (1995).
} 
CUADRO 8

RESULTADOS DE LOS CONTRASTES DE CAMBIO EN EL ORDEN DE INTEGRACIÓN

\begin{tabular}{|c|c|c|c|c|c|c|c|c|}
\hline & $\inf \left(t_{\rho 1}\right)$ & $\operatorname{media}\left(t_{\rho 1}\right)$ & $\inf \left(t_{\rho 2}\right)$ & $\operatorname{media}\left(t_{\rho 2}\right)$ & $\inf \left(t_{\gamma 1}\right)$ & $\operatorname{media}\left(t_{\gamma 1}\right)$ & $\inf \left(t_{\gamma^{2}}\right)$ & $\operatorname{media}\left(t_{\gamma 2}\right)$ \\
\hline \multirow[t]{2}{*}{ Alemania } & $-2,61$ & $-1,85$ & $-1,53$ & $-1,11$ & $-2,58$ & $-1,75$ & $-1,40$ & $-0,77$ \\
\hline & 1992 & - & 1975 & - & 1992 & - & 1968 & - \\
\hline \multirow[t]{2}{*}{ Austria } & $-2,13$ & $-1,59$ & $-1,74$ & $-0,83$ & $-2,13$ & $-1,60$ & $-1,76$ & $-0,89$ \\
\hline & 2003 & - & 1978 & - & 2003 & - & 1978 & - \\
\hline \multirow[t]{2}{*}{ Bélgica } & $-1,77$ & $-1,12$ & $-2,13$ & $-1,40$ & $-1,43$ & $-0,86$ & $-2,00$ & $-1,23$ \\
\hline & 1978 & - & 2003 & - & 1986 & - & 2003 & - \\
\hline \multirow[t]{2}{*}{ Dinamarca } & $-1,11$ & $-0,30$ & $-1,58$ & $-0,22$ & $-0,91$ & $-0,29$ & $-1,33$ & $-0,34$ \\
\hline & 2000 & - & 1987 & - & 2000 & - & 1987 & - \\
\hline \multirow[t]{2}{*}{ España } & $-4,48$ & $-3,46$ & $-2,82$ & $-2,40$ & $-3,89$ & $-2,85$ & $-2,57$ & $-1,41$ \\
\hline & 1999 & - & 1968 & - & 1999 & - & 1999 & - \\
\hline \multirow[t]{2}{*}{ Finlandia } & $-2,45$ & $-1,36$ & $-2,13$ & $-0,89$ & $-2,53$ & $-1,51$ & $-2,06$ & $-1,22$ \\
\hline & 1995 & - & 1976 & - & 1995 & - & 1976 & - \\
\hline \multirow[t]{2}{*}{ Francia } & $-2,93$ & $-2,16$ & $-1,60$ & $-0,61$ & $-2,96$ & $-2,18$ & $-1,69$ & $-0,56$ \\
\hline & 1995 & - & 1970 & - & 1995 & - & 1970 & - \\
\hline \multirow[t]{2}{*}{ Grecia } & $-2,62$ & $-1,70$ & $-0,88$ & $-0,73$ & $-2,56$ & $-1,59$ & $-0,76$ & $-0,38$ \\
\hline & 1996 & - & 1975 & - & 1996 & - & 2003 & - \\
\hline \multirow[t]{2}{*}{ Holanda } & $-2,74$ & $-2,28$ & $-2,27$ & $-1,98$ & $-2,16$ & $-1,54$ & $-1,72$ & $-1,01$ \\
\hline & 1993 & - & 1972 & - & 1999 & - & 1970 & - \\
\hline \multirow[t]{2}{*}{ Irlanda } & $-2,75$ & $-1,82$ & $-2,88$ & $-1,56$ & $-2,37$ & $-1,55$ & $-2,83$ & $-1,30$ \\
\hline & 1976 & - & 1982 & - & 1976 & - & 1982 & - \\
\hline \multirow[t]{2}{*}{ Italia } & $-3,33$ & $-2,40$ & $-2,53$ & $-0,68$ & $-3,36$ & $-2,45$ & $-2,58$ & $-0,75$ \\
\hline & 1994 & - & 1970 & - & 1994 & - & 1970 & - \\
\hline \multirow[t]{2}{*}{ Luxemburgo } & $-3,14$ & $-2,81$ & $-3,26$ & $-2,72$ & $-1,98$ & $-1,24$ & $-2,48$ & $-0,96$ \\
\hline & 1997 & - & 1968 & - & 2002 & - & 1970 & - \\
\hline \multirow[t]{2}{*}{ Portugal } & $-4,13$ & $-3,27$ & $-3,89$ & $-2,70$ & $-2,71$ & $-1,97$ & $-2,07$ & $-0,88$ \\
\hline & 1974 & - & 1974 & - & 1996 & - & 1983 & - \\
\hline \multirow[t]{2}{*}{ Suecia } & $-3,86$ & $-2,43$ & $-1,25$ & $-0,66$ & $-3,88^{*}$ & $-2,39$ & $-1,21$ & $-0,52$ \\
\hline & 1990 & - & 1970 & - & 1990 & - & 1974 & - \\
\hline \multirow[t]{2}{*}{ Reino Unido } & $-3,75$ & $-3,01$ & $-3,18$ & $-2,00$ & $-3,55$ & $-2,77$ & $-3,03$ & $-1,51$ \\
\hline & 1983 & - & 1970 & - & 1986 & - & 1975 & - \\
\hline V. cr. $5 \%$ & $-4,12$ & $-2,59$ & $-3,76$ & $-2,64$ & $-3,91$ & $-2,28$ & $-3,48$ & $-2,35$ \\
\hline
\end{tabular}

NOTAS: Valores críticos de FERNÁNDEZ (1999, Tabla III.1, pp. 108-109, t = 50). FUENTE: Elaboración propia.

\subsection{Contrastes de raíz unitaria no lineales}

Algunos estudios relativamente recientes analizan la hipótesis de solvencia exterior en distintos países, aplicando diversos test de raíces unitarias en un contexto nolineal, al ser considerado este como más apropiado a la hora de modelizar la evolución del saldo corriente. Ejemplos de estos trabajos son Chortareas y Kapetanios (2004), Kim et al. (2009), Christopoulos y León-Ledesma (2010) y Chen (2010).

Desde un punto de vista econométrico, si la serie de saldo por cuenta corriente presenta un comportamiento no-lineal, los test de raíces unitarias lineales general- 
mente aplicados sufren una pérdida importante de potencia, lo que puede llevar a aceptar la hipótesis de no solvencia aún no siendo cierta.

Con el fin de contemplar la posible no-linealidad del saldo por cuenta corriente en los países de la UE se ha procedido, en primer lugar, a contrastar dicha hipótesis a partir de los contrastes propuestos por Harvey et al. (2008) y Harvey y Leybourne (2007). A diferencia de otros contrastes, estos dos procedimientos permiten contrastar la hipótesis nula de linealidad cuando no se tiene certeza sobre el orden de integración de la serie en cuestión. A partir de los resultados mostrados en el Cuadro 9 se aprecia que en Austria, España, Finlandia, Francia, Irlanda y Luxemburgo, el saldo por cuenta corriente presenta un comportamiento no-lineal.

CUADRO 9

RESULTADOS DEL TEST DE LINEALIDAD

\begin{tabular}{|l|r|c|r|r|}
\hline & $\mathbf{W}_{\boldsymbol{\gamma}}$ & $\mathbf{W} * \mathbf{( 1 0} \%)$ & $\mathbf{W} * \mathbf{5 \%})$ & $\mathbf{W} * \mathbf{( 1 \% )}$ \\
\hline Alemania & 1,92 & 4,15 & 4,17 & 4,20 \\
Austria & 3,33 & 12,39 & 12,49 & 12,69 \\
Bélgica & 1,30 & 1,20 & 1,21 & 1,23 \\
Dinamarca & 0,36 & 2,53 & 2,61 & 2,74 \\
España & 6,09 & 13,81 & 13,87 & 13,99 \\
Finlandia & 11,51 & 10,72 & 10,81 & 10,98 \\
Francia & 8,84 & 10,64 & 10,74 & 10,91 \\
Grecia & 3,01 & 9,09 & 9,21 & 9,42 \\
Holanda & 4,14 & 2,50 & 2,52 & 2,55 \\
Irlanda & 2,15 & 11,00 & 11,07 & 11,20 \\
Italia & 3,04 & 5,44 & 5,49 & 5,58 \\
Luxemburgo & 7,85 & 17,43 & 17,52 & 17,68 \\
Portugal & 2,27 & 2,31 & 2,34 & 2,39 \\
Suecia & 2,67 & 4,27 & 4,29 & 4,32 \\
Reino Unido & 0,89 & 5,38 & 5,46 & 5,61 \\
Valor crítico 5\% & 5,99 & 7,78 & 9,49 & 13,28 \\
\hline
\end{tabular}

NOTAS: Los estadísticos $\mathrm{W}_{\gamma}$ (HARVEY et al., 2008) y W* (HARVEY y LEYBOURNE, 2007) se distribuyen como una chi-cuadrado con 2 y 4 grados de libertad respectivamente. FUENTE: Elaboración propia.

Para el conjunto de estos seis países, se ha procedido a aplicar el test KSS propuesto por Kapetanios et al. (2003) y el test KSS-MCG propuesto por Kapetanios y Shin (2008). Ambos tests permiten contrastar la hipótesis nula de presencia de raíz unitaria en un contexto no-lineal. Solo en el caso de Austria y Luxemburgo se encuentra evidencia a favor de la hipótesis de estacionariedad del saldo por cuenta corriente (Cuadro 10). 
CUADRO 10

RESULTADOS DEL TEST KSS Y KSS-GLS

\begin{tabular}{|l|c|c|c|c|c|}
\hline \multirow{2}{*}{} & \multicolumn{3}{|c|}{ KSS } & \multicolumn{2}{c|}{ KSS-GLS } \\
\cline { 2 - 6 } & $\boldsymbol{\tau}$ & $\boldsymbol{\tau}_{\boldsymbol{\mu}}$ & $\boldsymbol{\tau}_{\boldsymbol{\tau}}$ & $\boldsymbol{\tau}_{\boldsymbol{\mu}}$ & $\boldsymbol{\tau}_{\boldsymbol{\tau}}$ \\
\hline Austria & $-2,60$ & $-2,09$ & 1,32 & $-2,12$ & $-3,03$ \\
España & $-1,50$ & $-2,00$ & $-1,28$ & $-1,50$ & $-2,74$ \\
Finlandia & $-2,02$ & $-2,11$ & $-1,85$ & $-1,92$ & $-2,44$ \\
Francia & $-1,71$ & $-1,89$ & $-0,96$ & $-1,58$ & $-1,94$ \\
Irlanda & $-2,11$ & $-2,15$ & $-1,93$ & $-2,11$ & $-2,11$ \\
Luxemburgo & $-2,93$ & $-3,14$ & $-2,71$ & $-3,12$ & $-3,07$ \\
Valor crítico 5\% & $-2,22$ & $-2,93$ & $-3,40$ & $-2,21$ & $-2,93$ \\
\hline
\end{tabular}

NOTAS: Valores críticos de valores críticos han sido obtenidos de KAPETANIOS et al. (2003) y KAPETANIOS y SHIN (2008). El número de retardos $(P)$ incluidos en cada estimación ha sido elegido mediante el criterio de información CS propuesto por SCHWARZ (1978).

FUENTE: Elaboración propia.

Una vez presentados los resultados de los distintos contrastes aplicados se puede concluir que los países en donde el saldo por cuenta corriente presenta un comportamiento estacionario, compatible con el concepto de solvencia exterior son: Austria, Grecia, Holanda, Luxemburgo, Reino Unido y Suecia. Por su parte, en el conjunto de países formado por Alemania, Bélgica, Dinamarca, España, Francia, Irlanda, Italia y Portugal, la evidencia no es favorable al concepto de solvencia. En el caso de Finlandia, los resultados no son del todo concluyentes. Esta clasificación sin embargo requiere ser matizada, pues la situación de los países que conforman cada uno de los dos grupos dista mucho de ser homogénea. Así, dentro del conjunto de países donde la evidencia es favorable a la solvencia exterior, el caso de Grecia precisa de una cierta reflexión. Si bien los contrastes aplicados parecen ser favorables al cumplimiento de la RPI, el hecho de que el déficit corriente promedio durante los últimos doce años se sitúe cercano al 13 por 100, pone en duda que dicha situación pueda sostenerse de forma indefinida en el tiempo. En una posición mucho más moderada se encuentra el Reino Unido, en donde el déficit deambula, al menos durante las últimas dos décadas, en el entorno del 2 por 100. En contraposición a estos dos casos, Austria, Holanda y Suecia son países en donde no sólo la evolución del saldo a lo largo del periodo muestral es compatible con la RPI, sino que además presentan un saldo favorable durante la última década. Un análisis similar dentro del conjunto de países donde la evidencia no es favorable al concepto de solvencia exterior permite hacer las siguientes distinciones. La situación de Alemania, Bélgica y Dinamarca difiere notablemente de la del grupo de países formado por España Francia, Italia y Portugal. Pues en efecto, mientras que en los primeros se mantiene un saldo favorable, en los segundos el resultado de la cuenta corriente se ha ido deteriorando de forma continuada desde finales de los años noventa hasta posicionarse en una situación que posiblemente no pueda sostenerse indefinidamente. 


\section{Conclusiones}

La Unión Europea ha mantenido hasta años muy cercanos un comportamiento en exceso complaciente respecto a los déficits exteriores, medidos por el saldo de la cuenta corriente. Sin embargo, la evidencia empírica acumulada desde comienzos de los años noventa del siglo pasado y, especialmente, la extraída de los años recientes de crisis, muestran que los problemas exteriores necesitan atención preferente entre los políticos y académicos. Esto es consecuencia de la evidente polarización de los resultados que se registra en los momentos presentes en los países de la UE-15.

En este trabajo, se ha examinado el problema de la solvencia, entendida como cumplimiento de la RPI y medida por la estacionariedad del saldo de la cuenta corriente de los diversos países. Dando un paso más, se ha cualificado el resultado derivado del análisis de estacionariedad con la información sobre las magnitudes relevantes de la economía, con el fin de analizar si la situación de determinados países es, además de solvente, en términos de RPI, sostenible, es decir, si se van a poder mantener aquellas condiciones sobre las que se basa la solvencia o previsiblemente no se van a poder mantener y, más pronto o más tarde, se ha de provocar un giro en la situación.

Cabe destacar que, entre los países que aparecen como solventes, según la RPI, el caso de Grecia debe ser matizado fuertemente, puesto que las informaciones económicas apuntan con claridad a que esta situación de solvencia será difícilmente sostenible en el futuro sin cambios importantes en sus pautas de comportamiento.

Dentro del grupo de países que aparecen incumpliendo la RPI, al haber síntomas de no estacionariedad de la cuenta corriente, también es importante matizar la diversidad de casos, como por ejemplo, los de Alemania y Dinamarca, cuya situación de no estacionariedad apuntaría a la supersolvencia, y España y Portugal, cuyo incumplimiento derivaría de una situación de previsible insolvencia.

En el caso particular de España, los resultados obtenidos indican, de modo resumido, lo siguiente: el análisis de estacionariedad de la serie de saldo por cuenta corriente para el periodo 1960-2010 a partir de la aplicación de tests de raíces unitarias tradicionales, permite confirmar la solvencia del sector exterior español. Cuando se tiene en cuenta la posible presencia de cambio estructural en la tendencia estocástica de la serie se mantiene este resultado, si bien el cambio estructural no resulta estadísticamente significativo. Además, se encuentra evidencia de la presencia de un cambio en el orden de integración del saldo de manera que entre 1960 y 1998 el saldo presenta un comportamiento estacionario, si bien a partir de 1999 pasa a ser no estacionario. Este resultado resulta interesante puesto que parece indicar que la evidencia a favor de la no solvencia exterior de la economía española se debe al continuo deterioro del saldo corriente a partir de la incorporación de España a la zona del Euro. Teniendo en cuenta que la evolución del saldo en el periodo considerado puede ser no-lineal, la evidencia en contra del cumplimiento de la RPI se mantiene. 


\section{Bibliografía}

[1] AHMED, S. y ROGERS, J. H. (1995): «Government budget deficits and trade deficits. Are present value constraints satisfied in long term data?», Journal of Monetary Economics, vol. 36, pp. 351-374.

[2] ANCHUELO, A. (1995): «Sostenibilidad del déficit exterior español», Información Comercial Española, vol. 742, pp. 127-139.

[3] APERGIS, N.; KATRAKILIDIS, K. P. y TABAKIS, N. M. (2000) «Current Account Deficit Sustainability: The Case of Greece», Applied Economics Letters, vol.7, pp. 599603.

[5] ARESTIS, P. H. y PAÚL GUTIÉRREZ, J. (2009): «Déficits en cuenta corriente en la Unión Económica y Monetaria y crisis financiera internacional», Ola Financiera, n. ${ }^{\circ} 4$, septiembre-diciembre, pp. 2-42.

[6] BITZIS, G.; PALEOLOGOS, J. M. y PAPAZOGLOU, C. (2008): «The determinants of the greek current account deficit: the EMU experience», Journal of International and

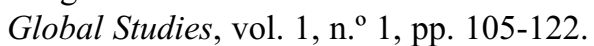

[7] CHEN, S. W. (2010): «Testing for the sustainability of the current account deficit in four industrial countries: a revisitation», Economics Bulletin, 30(2).

[8] CHEN, S. W. (2011a): «Current account deficits and sustainability: evidence from the OECD countries», Economic Modelling, vol. 28, pp. 1455-1464.

[9] CHEN, S. W. (2011b): «Are current account deficits really sustainable in the G-7 countries?», Japan and the World Economy, vol. 23, pp. 190-201.

[10] CHORTAREAS, G. E. y KAPETANEOS, G. (2004): «An investigation of current solvency in Latin America using non-linear nonstationarity tests», Studies in Non-Linear Dynamics and Econometrics, 8(4).

[11] CHRISTIANO, L. J. (1992): «Searching for a break in GNP», Journal of Business and Economic Statistics, 10, 237-250.

[12] CHRISTOPOULUS, D. K. y LEON-LEDESMA, M. (2010): «Current-account sustainability in the US: what do we really know about it?», Journal of International Money and Finance, 29, 442-459.

[13] CLARIDA, R. H.; GORETTI, M. y TAYLOR, M. P. (2007): «Are there Thresholds of Current Account Adjustment in The G-7?», en R. H. CLARIDA (ed.), G7 Current Account Imbalances: Sustainability and Adjustment, University of Chicago Press.

[14] DICKEY, D. A. y FULLER, W. A. (1979): «Distribution of the estimators for autoregressive time series with a unit root», Journal of the American Statistical Association, vol. 74, pp. 427-431.

[15] DICKEY, D. A. y FULLER, W. A. (1981): «Likelihood ratio statistics for autoregressive time series with a unit root», Econometrica, vol. 49, pp. 1057-1072.

[16] DONOSO, V. y FARIÑAS, J. C. (2011); «El sector exterior», cap. 13 de J. L. García Delgado y R. Myro, Lecciones de Economía Española, Thomson Reuters, Madrid, décima edición.

[17] DÜLGER, F. y OZDEMIR, Z. A. (2005): «Current account sustainability in seven developed countries», Journal of Economics and Social Research, vol. 7, pp. 47-80.

[18] ELLIOT, G.; ROTHENBERG, T. y STOCK, J. (1996): «Efficient test for an autoregressive unit root», Econometrica, vol. 64, pp. 813-836.

[19] FERNÁNDEZ CASTRO, B. (2002): «La deuda exterior, su sostenibilidad y la solvencia de la economía española desde una perspectiva intertemporal», tesis doctoral, 
Departamento de Fundamentos del Análisis Económico, Universidad de Santiago de Compostela.

[20] FERNÁNDEZ, E. (2007): «La sostenibilidad del déficit exterior de los Estados Unidos», Documentos de Economía 'la Caixa', n. ${ }^{\circ}$ 5, abril.

[21] FERNÁNDEZ, J. L. (1999): «Efecto de los cambios estructurales en el análisis de series económicas no estacionarias», Universidad Complutense de Madrid, mimeo.

[22] FERNÁNDEZ, J. L. y PERUGA, R. (2005): «Un contraste ADF secuencial para la detección de cambios en el orden de integración», Revista de Economía Aplicada, 37, 107-137.

[23] FONDO MONETARIO INTERNACIONAL (1999): «Denmark: selected issues», IMF Staff Country Report, n. ${ }^{\circ}$ 99/107.

[24] FISHER, E. (1995): «A new way to think about the current account», International Economic Review, vol. 36, pp. 555-568.

[25] GUNDLACH, E. y SINN, S. (1992): «Unit Root Tests of the Current Account Balance: Implications for International Capital Mobility», Applied Economics, vol. 24, pp. $617-25$.

[26] HARRIS, D.; MCCABE, B. P. y LEYBOURNE, S. J. (2003): «Some limit theory for autocovariances whose order depends on sample size», Econometric Theory, vol. 19, pp. 829-864.

[27] HARVEY, D. I. y LEYBOURNE, S. J. (2007): «Testing for time series linearity», Econometrics Journal, vol. 10, pp. 149-165.

[28] HARVEY, D. I.; LEYBOURNE, S. J. y XIAO, B. (2008): «A powerful test for linearity when the order of integration is unknown», Studies in Nonlinear Dynamics and Econometrics, vol. 12, n. ${ }^{\circ} 3$.

[29] HUSTED, S. (1992): «The emerging U.S. current account deficit in the 1980s: a cointegration analysis», The Review of Economics and Statistics, vol. 74, n. ${ }^{\circ}$ 1, pp. 159-166.

[30] HOLMES, M. J. (2006): «How sustainable are OECD current account balances in the long-run?, Manchester School, vol. 74, pp. 626-643.

[31] JONUNG, L. y HAGBERG, T. (2005): «How costly was the crisis of the 1990s? A comparative analysis of the deepest crises in Finland and Sweden over the last 130 years», Economic Paper, n. ${ }^{\circ}$ 224, Comisión Europea.

[32] KALYONCU, H. (2006): «International intertemporal solvency in OECD countries: evidence from panel unit root», Prague Economic Papers, vol. 1, pp. 44-49

[33] KAPETANIOS, G. y SHIN, Y. (2008): «GLS detrending-based unit root tests in nonlinear STAR and SETAR models», Economics Letters, 100, 377-380.

[34] KAPETANIOS, G.; SNELL, A. y SHIN, Y. (2003): «Testing for a unit root in the nonlinear STAR framework», Journal of Econometrics, 112, 359-379.

[35] KIM, B.; MIN, H. G. y McDONALD, J. A. (2009): "Are asian countries' current accounts sustainable? Deficits, even when associated with high investment, are not costless», Journal of Policy Modelling, 31, 163-179.

[36] LEACHMAN, L. L. y FRANCIS, B. (2000): «Multicointegration análisis of the sustainability of foreign debt», Journal of Macroeconomics, vol. 22, n. ${ }^{\circ}$ 2, pp. 207-227.

[37] LIU, T. C. y TANNER, E. (1996): «International Intertemporal Solvency in Industrialized Countries: Evidence and Implications», Southern Economic Journal, vol. 62, n. ${ }^{\circ} 3$, pp. $739-749$.

[38] MACKINNON, J. (1996): «Numerical distribution functions for unit root and cointegration tests», Journal of Applied Econometrics, vol. 11, pp. 601-618. 
[39] MILESI-FERRETTI, G. M. y RAZIN, A. (1999): «Current-Account Sustainability», Princeton Studies in International Finance, n. ${ }^{\circ}$ 81, Department of Economics, Princeton University, octubre.

[40] OTTO, G. (1992): «Testing a present value model of current account: evidence from US and canadian time series», Journal of International Money and Finance, vol. 11, pp. 414-430.

[41] RAYBADUI, M.; SOLA, M. y SPAGNOLO, F. (2004): «Red Signals: Current Account Deficits and Sustainability», Economic Letters, vol. 84, pp. 217-223.

[42] SCHWARZ, G. (1978): «Estimating the dimension of a model», The Annals of Statistics, vol. 6, pp. 461-471.

[43] TREHAN, B. y WALSH, C. (1991): «Testing intertemporal budget constraints: teory and application to US Federal Budget deficits and current account deficits», Journal of Money, Credit and Banking, vol. 23, n. ${ }^{\circ}$ 2. pp. 206-223.

[44] WICKENS, M. R. y UCTUM, M. (1993): «The Sustainability of Current Account Deficits: A Test of the U.S. Intertemporal Budget Constraint», Journal of Economic Dynamics and Control, vol. 17, pp. 423-441.

[45] WU, J. L.; FOUNTAS,, S. y CHEN, S. L. (1996): «Testing for the sustainability of current account deficit in two industrial countries», Economics Letters, vol. 52, pp. 193 198.

[46] WU, J.L. (2000): «Mean Reversion of the Current Account: Evidence from Panel Data Unit- Root Test», Economic Letters, vol. 66, pp. 215-222.

[47] WU, J .L.; CHEN, S. L. y LEE, H. Y. (2001): «Are current account deficits sustainable? Evidence from panel cointegration», Economics Letters, vol. 72, pp. 219-224.

[48] ZIVOT, E. y ANDREWS, D. (1992): «Further evidence on the great crash, the oil price shock, and the unit root hypothesis», Journal of Business and Economic Statistics, 10, 251-270. 


\section{APÉNDICE}

\section{Test de raíces unitarias DFA}

El test se obtiene a partir del estadístico $t$ de Student para el contraste de la hipótesis nula $\mathrm{H}_{0}: \rho=0$ (raíz unitaria) frente a la hipótesis alternativa $\mathrm{H}_{1}: \rho<0$ mediante la estimación, por Mínimos Cuadrados Ordinarios (MCO), de la siguiente ecuación:

$$
\Delta C A_{t}=\alpha+\beta t+\rho C A_{t-1}+\sum_{i=1}^{P} \delta_{i} \Delta C A_{t-i}+\varepsilon_{t}
$$

en donde $\Delta=(1-B)$, siendo $B$ el operador de retardos. El modelo permite tres especificaciones alternativas en cuanto a los componentes deterministas se refiere: modelo sin componentes deterministas $(\tau: \alpha=\beta=0)$, modelo con constante $\left(\tau_{u}: \beta=0\right)$, y modelo con constante y tendencia $\left(\tau_{\tau}\right)$.

\section{Test de raíces unitarias DF-MCG}

El contraste se realiza estimando por MCO, en primer lugar, la ecuación en cuasidiferencias,

$$
d\left(C A_{t} \mid a\right)=d\left(X_{t} \mid a\right)^{\prime} \phi(a)+u_{t}
$$

en donde $d\left(z_{t} \mid a\right)=z_{t}$ si $t=1$ y d $\left(z_{t} \mid a\right)=z_{t}-a z_{t-1}$ para $t>1$, y $X_{t}$ incluye bien una constante, bien una constante y tendencia. Los autores recomiendan fijar a $=1-7 / \mathrm{T}$ si $X_{t}$ incluye sólo una constante y a $=13,5 / T$ si incluye además una tendencia lineal. En segundo lugar se aplica el test DFA sobre la serie $\mathrm{CA}_{\mathrm{t}}^{\mathrm{d}}$ obtenida a partir de:

$$
C A_{t}^{d} \equiv C A_{t}-X_{t}^{\prime} \hat{\phi}(a)
$$

Los resultados del contraste se presentan para el caso en el que se incluye una constante $\left(\tau_{\mu}\right)$ y una constante y tendencia $\left(\tau_{\tau}\right)$.

\section{Cambio estructural en la tendencia estocástica}

La metodología propuesta por Fernández (1999) y Fernández y Peruga (2005) consiste en la aplicación secuencial del test DFA sobre la siguiente ecuación,

$$
\Delta C A_{t}=\alpha+\alpha^{\prime} D_{\lambda t}+\rho C A_{t-1}+\sum_{i=1}^{P} \delta_{i} \Delta C A_{t-i}+\varepsilon_{t}
$$


en donde:

$$
D_{\lambda t}=\left\{\begin{array}{ll}
0 & \text { si } t<\tau \\
1 & \text { si } t \geq \tau
\end{array} \quad, \tau \in[0,15 T, 0,85 T]\right.
$$

es una variable ficticia que introduce el cambio estructural en el intervalo comprendido entre el 15 y el 85 por 100 del periodo muestral disponible. Para cada posible punto de cambio estructural dentro del intervalo previamente definido, se calculan tres estadísticos: (i) el estadístico DFA $\left(\mathrm{t}_{\rho}\right)$ para el contraste de presencia de raíz unitaria $(\rho=0)$, (ii) el valor absoluto del estadístico $t$ de Student $\left(\mid \mathrm{t}_{\alpha^{\prime}}\right)$ para el contraste de la hipótesis nula $\alpha^{\prime}=0$, y (iii) el valor absoluto del estadístico $t$ de Student $\left(\left|t_{\left(\alpha^{\prime}\right)}\right|\right)$ para el contraste de la hipótesis nula $\alpha^{\prime}=0$ imponiendo el supuesto de presencia de raíz unitaria $(\rho=1)$ en [1.11]. Para cada una de las secuencias obtenidas de estos tres estadísticos se calcula el valor medio y el ínfimo o supremo según el caso, de manera que contamos con seis estadísticos de contraste: $\inf \left(\mathrm{t}_{\rho}\right)$, media $\left(\mathrm{t}_{\rho}\right)$, $\sup \left(\left|\mathrm{t}_{\alpha^{\prime}}\right|\right)$, media $\left(\mid \mathrm{t}_{\alpha^{\prime}}\right), \sup \left(\mid \mathrm{tt}_{\left(\alpha^{\prime}\right)}\right)$, y media $\left(\mathrm{It}_{\left(\alpha^{\prime}\right)} \mid\right)$. Los dos primeros estadísticos permiten contrastar la presencia de raíz unitaria bajo la presencia de cambio estructural, entendido este como un cambio en el término constante del modelo. Por su parte, los estadísticos $\sup \left(\left|\mathrm{t}_{\alpha^{\prime}}\right|\right)$ y media $\left(\left|\mathrm{t}_{\alpha^{\prime}}\right|\right)$ y los estadísticos $\sup \left(\left|\mathrm{t}_{\left(\alpha^{\prime}\right)}\right|\right)$ y media $\left(\left|\mathrm{t}_{\left(\alpha^{\prime}\right)}\right|\right)$ permiten contrastar la significatividad del cambio estructural en un contexto en donde el saldo por cuenta corriente es un proceso estacionario y no estacionario respectivamente.

\section{Cambio en el orden de integración}

La metodología propuesta por Fernández (1999) y Fernández y Peruga (2005) para analizar posibles cambios en el orden de integración de una serie consiste en la estimación secuencial de las siguientes ecuaciones,

$$
\begin{gathered}
\Delta C A_{t}=\alpha+\rho_{1}\left[1-D_{\lambda t}\right] C A_{t-1}+\rho_{2} D_{\lambda t} C A_{t-1}+\sum_{i=1}^{P} \delta_{i} \Delta C A_{t-i}+\varepsilon_{t} \\
\Delta C A_{t}=\alpha+\gamma_{1}\left[1-D_{\lambda t}\right] C A_{t-1}+\sum_{i=1}^{P} \delta_{i} \Delta C A_{t-i}+\varepsilon_{t} \\
\Delta C A_{t}=\alpha+\gamma_{2} D_{\lambda t} C A_{t-1}+\sum_{i=1}^{P} \delta_{i} \Delta C A_{t-i}+\varepsilon_{t}
\end{gathered}
$$

en donde $\mathrm{D}_{\gamma \mathrm{t}}$ viene definido por [1.12]. La estimación secuencial de [1.13], para cada una de las observaciones del intervalo comprendido entre el 15 y 85 por 100 del periodo muestral, permite contrastar la existencia de raíz unitaria en las dos partes en que se divide la muestra a partir del estadístico $t$ de Student para el contraste de la hipótesis nula $\rho_{1}=0\left(\mathrm{t}_{\rho 1}\right)$ y $\rho_{2}=0\left(\mathrm{t}_{\rho 2}\right)$. En las otras dos ecuaciones se con- 
trasta la presencia de raíz unitaria en una sola parte de la muestra. La estimación de [1.14] y [1.15] permiten calcular el estadístico $t$ de Student para el contraste de la hipótesis nula de presencia de raíz unitaria en la primera parte $\left(t_{\gamma 1}, H_{0}: \gamma_{1}=0\right)$ y en la segunda parte de la muestra $\left(t_{\gamma 2}, H_{0}: \gamma_{2}=0\right)$ respectivamente. En ambos casos se impone la restricción de que la parte complementaria de la muestra presenta un comportamiento no estacionario. Una vez obtenida la secuencia para los cuatro estadísticos considerados, se calcula el ínfimo y la media: $\inf \left(\mathrm{t}_{\rho 1}\right)$, media $\left(\mathrm{t}_{\rho 1}\right)$, $\inf \left(\mathrm{t}_{\rho 2}\right), \operatorname{media}\left(\mathrm{t}_{\rho 2}\right), \inf \left(\mathrm{t}_{\gamma 1}\right), \operatorname{media}\left(\mathrm{t}_{\gamma 1}\right), \inf \left(\mathrm{t}_{\gamma 2}\right)$ y media $\left(\mathrm{t}_{\gamma 2}\right)$.

\section{Contrastes de linealidad}

El procedimiento propuesto por Harvey y Leybourne (2007) parte de la especificación de la siguiente ecuación:

$$
C A_{t}=\alpha+\sum_{i=1}^{3} \beta_{i} C A_{t-1}^{i}+\sum_{i=1}^{3} \delta_{i} \Delta C A_{t-1}^{i}+\varepsilon_{t}
$$

La estimación por MCO de la ecuación sin restringir y la estimación restringida para $\beta_{2}=\beta_{3}=\delta_{2}=\delta_{3}=0$, permiten obtener el siguiente estadístico de Wald a partir de la suma residual restringida (SRR) y sin restringir (SRS):

$$
W=\frac{S R R-S R S}{S R S / T}
$$

El estadístico para el contraste de la hipótesis nula de linealidad se calcula como:

$$
W^{*}=\exp \left(-b|D F A|^{-1}\right) W
$$

en donde DFA es el valor del estadístico Dickey-Fuller Aumentado. Los autores muestran que el estadístico $\mathrm{W}^{*}$ se distribuye como una chi-cuadrado con 4 grados de libertad.

Por su parte, la metodología propuesta por Harvey et al. (2008) parte de la estimación por MCO de las dos siguientes ecuaciones:

$$
\begin{gathered}
C A_{t}=\alpha+\sum_{i=1}^{3} \beta_{i} C A_{t-1}^{i}+\sum_{i=1}^{P} \delta_{i} \Delta C A_{t-1} \\
\Delta C A_{t}=\alpha+\sum_{i=1}^{3} \beta_{i} \Delta C A_{t-1}^{i}+\sum_{i=1}^{P} \delta_{i} \Delta C A_{t-1}+\varepsilon_{t}
\end{gathered}
$$


En segundo lugar se calculan los siguientes estadísticos de Wald, $\mathrm{W}_{0} \mathrm{y} \mathrm{W}_{1}$, a partir de [1.19] y [1.20] respectivamente:

$$
\begin{gathered}
W_{0}=\frac{S R R_{0}-S R S_{0}}{S R S_{0} / T} \\
W_{1}=\frac{S R R_{1}-S R S_{1}}{S R S_{1} / T}
\end{gathered}
$$

en donde $\mathrm{SRR}_{0}$ y $\mathrm{SRR}_{1}$ es la suma residual restringida para $\beta_{2}=\beta_{3}=0$ en [1.19] y [1.20], respectivamente, y donde $\mathrm{SRS}_{0}$ y $\mathrm{SRS}_{1}$ es la suma residual sin restringir. El estadístico para el contraste de la hipótesis nula de linealidad se calcula como:

$$
W_{\lambda}=\left\{1-\exp \left(-g\left(\frac{D F A}{S}\right)^{2}\right)\right\} W_{0}+\exp \left(-g\left(\frac{D F A}{S}\right)^{2}\right) W_{1}
$$

en donde en donde DFA es el valor del estadístico Dickey-Fuller aumentado y $\mathrm{S}$ es el valor del estadístico no paramétrico de estacionariedad propuesto por Harris et al. (2003). 